\title{
Two-Higgs-doublet-portal dark-matter models in light of direct search and LHC data
}

\author{
Chia-Feng Chang, ${ }^{a}$ Xiao-Gang $\mathbf{H e}^{a, b, c}$ and Jusak Tandean ${ }^{a, c}$ \\ ${ }^{a}$ Department of Physics and Center for Theoretical Sciences, National Taiwan University, \\ No. 1, Sec. 4, Roosevelt Rd., Taipei 106, Taiwan \\ ${ }^{b}$ INPAC, Department of Physics and Astronomy, Shanghai Jiao Tong University, \\ 800 Dongchuan Rd., Minhang, Shanghai 200240, China \\ ${ }^{c}$ Physics Division, National Center for Theoretical Sciences, \\ No. 101, Sec. 2, Kuang Fu Rd., Hsinchu 300, Taiwan \\ E-mail: a29788685@gmail.com, hexg@phys.ntu.edu.tw, jtandean@yahoo.com
}

ABSTRACT: We explore simple Higgs-portal models of dark matter (DM) with spin 1/2, $3 / 2$, and 1, respectively, applying to them constraints from the LUX and PandaX-II direct detection experiments and from LHC measurements on the $125-\mathrm{GeV}$ Higgs boson. With only one Higgs doublet, we find that the spin-1/2 DM having a purely scalar effective coupling to the doublet is viable only in a narrow range of mass near the Higgs pole, whereas the vector DM is still allowed if its mass is also close to the Higgs pole or exceeds 1.4 TeV, both in line with earlier analyses. Moreover, the spin-3/2 DM is in a roughly similar situation to the spin- $1 / 2 \mathrm{DM}$, but has surviving parameter space which is even more restricted. We also consider the two-Higgs-doublet extension of each of the preceding models, assuming that the expanded Yukawa sector is that of the two-Higgs-doublet model of type II. We show that in these two-Higgs-doublet-portal models significant portions of the DM mass regions excluded in the simplest scenarios by direct search bounds can be reclaimed due to suppression of the effective DM interactions with nucleons at some ratios of the $C P$-even Higgs bosons' couplings to the up and down quarks. The regained parameter space contains areas which can yield a DM-nucleon scattering cross-section that is far less than its current experimental limit or even goes below the neutrino-background floor.

KeYwords: Beyond Standard Model, Cosmology of Theories beyond the SM, Effective Field Theories, Higgs Physics

ARXIV EPRINT: 1702.02924 


\section{Contents}

1 Introduction 1

2 Minimal Higgs-portal fermionic and vector DM models 2

2.1 Spin- $1 / 2$ dark matter 2

2.2 Spin-3/2 dark matter 5

$\begin{array}{lll}2.3 & \text { Vector dark matter } & 7\end{array}$

3 Two-Higgs-doublet-portal fermionic and vector DM models $\quad 8$

$\begin{array}{lll}3.1 & \text { THDM II }+\psi & 12\end{array}$

$\begin{array}{lll}3.2 & \text { THDM II }+\boldsymbol{\Psi} & 15\end{array}$

$\begin{array}{lll}3.3 & \text { THDM II }+\mathrm{V} & 17\end{array}$

$\begin{array}{llr}4 & \text { Conclusions } & 19\end{array}$

A Extra formulas for DM reactions $\quad 20$

B Conditions for perturbativity, vacuum stability, and tree-level unitarity 22

\section{Introduction}

The latest direct searches for weakly interacting massive particle (WIMP) dark matter (DM) by the LUX and PandaX-II Collaborations [1, 2] have turned up null results, leading to the strictest upper-limits to date on the cross section of spin-independent elastic WIMPnucleon scattering in the mass region from about $4 \mathrm{GeV}$ to $100 \mathrm{TeV}$. For lower WIMP masses down to $0.5 \mathrm{GeV}$, the existing corresponding limits were set a little earlier in the CRESST [3] and CDMSlite [4] experiments. Ongoing and planned efforts to detect the DM directly [5] will likely improve upon these findings if it still eludes discovery.

The above recent measurements translate into substantial restrictions on WIMP DM models, especially minimal Higgs-portal ones, which are also subject to constraints from continuing quests at the LHC [6-8] for decays of the 125-GeV Higgs boson into final states that would signal new physics beyond the standard model (SM). The combination of restraints from direct detection and LHC data has ruled out in particular the DM mass region below $0.5 \mathrm{TeV}$ or so in the simplest Higgs-portal real-scalar-DM model, with the exception of a narrow range around the Higgs pole [9-13]. If the DM is instead a spin- $1 / 2$ fermion with a purely scalar effective coupling to the Higgs, only this small region near the Higgs pole remains viable [10, 14].

Nevertheless, as previously demonstrated in the Higgs-portal scalar-DM case [9, 1519], by adding another Higgs doublet to the simplest scenario it is possible to decrease 
the effective interactions of the DM with nucleons sufficiently and thereby to regain at least some of the parameter space disallowed by the direct searches. This motivates us to explore similar ideas in other simple models. Specifically, in this paper we first revisit the minimal Higgs-portal scenarios in which the DM is a fermion of spin $1 / 2$ or $3 / 2$ or a spin-1 boson, to see how the aforesaid restraints impact them. Subsequently, we consider a somewhat expanded version of each of the models by incorporating another Higgs doublet and arranging the new Yukawa sector to be that of the so-called two-Higgs-doublet model (THDM) of type II. We will show that these two-Higgs-doublet-portal DM models, like their scalar-DM counterpart, potentially have ample parameter space that can avoid all the latest direct-detection limits and may even evade future ones. It is worth mentioning here that there have been a number of studies in the past on various Higgs-portal scenarios in which a THDM was supplemented with a SM-gauge-singlet DM candidate having spin 0 [15-32], $1 / 2$ [31-33], or 1 [32].

The organization of the rest of the paper is as follows. In section 2 we take another look at the available constraints from DM direct searches and LHC Higgs measurements on the three different minimal Higgs-portal models having WIMP DM candidates with spin $1 / 2,3 / 2$, and 1 , respectively. In section 3 , we deal with the two-Higgs-doublet extensions of these models where the enlarged Yukawa sector is that of the type-II THDM. We will address how the extended models can escape some of the restrictions in the presence of sizable breaking of isospin symmetry in the DM effective interactions with nucleons. ${ }^{1}$ We give our conclusions in section 4 . We collect additional formulas and extra details in a couple of appendices.

\section{Minimal Higgs-portal fermionic and vector DM models}

\subsection{Spin-1/2 dark matter}

In the most economical fermionic scenario [44], the SM is slightly enlarged by the inclusion of a spin-1/2 Dirac field $\psi$ which is a singlet under the SM gauge group and serves as the WIMP DM candidate. In this model, hereafter referred to as $\mathrm{SM}+\psi$, the $\mathrm{DM}$ is stable due to an exactly preserved $Z_{2}$ symmetry under which $\psi \rightarrow-\psi$, while the SM fields are unchanged. Since $\psi$ cannot couple directly to SM members in a renormalizable way, without explicitly introducing other new ingredients one can explore $\psi$ interactions with the SM sector that are induced by effective nonrenormalizable operators. The simplest ones with a Higgs doublet $\mathrm{H}$ are the dimension-five combinations $\bar{\psi}\left(1 \pm \gamma_{5}\right) \psi \boldsymbol{H}^{\dagger} \mathrm{H}$, which are invariant under the SM gauge group. Assuming that the Lagrangian $\mathcal{L}_{\psi}$ for $\psi$ conserves $C P$ symmetry, making the pseudoscalar coupling absent, one can then write [44]

$$
\mathcal{L}_{\psi}=\bar{\psi} i \not \partial \psi-\mu_{\psi} \bar{\psi} \psi-\frac{\bar{\psi} \psi \mathrm{H}^{\dagger} \mathrm{H}}{\Lambda_{\psi}}
$$

\footnotetext{
${ }^{1}$ Isospin violation in DM interactions can occur not only in a THDM plus SM-singlet DM [9, 15-33], but also in certain other models, such as those in which the DM couples to a $Z^{\prime}$ boson [34-37]. More general aspects of isospin-violating DM have been discussed in [38-43].
} 
where $\mu_{\psi}$ and $\Lambda_{\psi}$ are real constants of dimension mass and $\Lambda_{\psi}$ absorbs the parameters of the underlying heavy physics. ${ }^{2}$ After electroweak symmetry breaking, $\mathrm{H}^{\dagger} \mathrm{H}=\frac{1}{2}(h+v)^{2}$, and so

$$
\mathcal{L}_{\psi} \supset-m_{\psi} \bar{\psi} \psi-\lambda_{\psi h} \bar{\psi} \psi\left(h+\frac{h^{2}}{2 v}\right), \quad m_{\psi}=\mu_{\psi}+\frac{\lambda_{\psi h} v}{2}, \quad \lambda_{\psi h}=\frac{v}{\Lambda_{\psi}},
$$

where $h$ is the physical Higgs and $v \simeq 246 \mathrm{GeV}$ the vacuum expectation value (VEV) of $\mathrm{H}$. The DM mass $m_{\psi}$ and the DM-Higgs coupling $\lambda_{\psi h}$ are the only free parameters in $\mathcal{L}_{\psi}$.

The $\lambda_{\psi h}$ terms in eq. (2.2) are responsible for the DM relic density. It results from $\bar{\psi} \psi$ annihilation into SM particles, which happens mainly via the Higgs-exchange process $\bar{\psi} \psi \rightarrow h^{*} \rightarrow X_{\mathrm{SM}}$. If the $\bar{\psi} \psi$ pair has a center-of-mass (c.m.) energy $\sqrt{s}>2 m_{h}$, the channel $\bar{\psi} \psi \rightarrow h h$ needs to be taken into account. Thus, we can express the cross section $\sigma_{\text {ann }}$ of DM annihilation as

$$
\begin{aligned}
\sigma_{\mathrm{ann}} & =\sigma\left(\bar{\psi} \psi \rightarrow h^{*} \rightarrow X_{\mathrm{SM}}\right)+\sigma(\bar{\psi} \psi \rightarrow h h) \\
\sigma\left(\bar{\psi} \psi \rightarrow h^{*} \rightarrow X_{\mathrm{SM}}\right) & =\frac{\beta_{\psi} \lambda_{\psi h}^{2} \sqrt{s} \sum_{i} \Gamma\left(\tilde{h} \rightarrow X_{i, \mathrm{SM}}\right)}{2\left[\left(m_{h}^{2}-s\right)^{2}+\Gamma_{h}^{2} m_{h}^{2}\right]}, \quad \beta_{\mathrm{x}}=\sqrt{1-\frac{4 m_{\mathrm{x}}^{2}}{s}}
\end{aligned}
$$

where the formula for $\sigma(\bar{\psi} \psi \rightarrow h h)$ is relegated to appendix A, the sum in the second line is over SM final-states $X_{i, \mathrm{SM}} \neq h h$, and $\tilde{h}$ refers to a virtual Higgs having a mass $m_{\tilde{h}}=\sqrt{s}$. Subsequently, we can extract $\lambda_{\psi h}$ from the observed DM abundance, as outlined in appendix A, and then test the result with various constraints.

One of the important restrictions on $\lambda_{\psi h}$ applies in the region $m_{\psi}<m_{h} / 2$, where the invisible decay channel $h \rightarrow \bar{\psi} \psi$ is open and contributes to the Higgs' total width $\Gamma_{h}=\Gamma_{h}^{\mathrm{SM}}+\Gamma(h \rightarrow \bar{\psi} \psi)$. From eq. (2.2), we obtain the partial rate

$$
\Gamma(h \rightarrow \bar{\psi} \psi)=\frac{\lambda_{\psi h}^{2} m_{h}}{8 \pi}\left(1-\frac{4 m_{\psi}^{2}}{m_{h}^{2}}\right)^{3 / 2} .
$$

The experiments at the LHC offer information pertaining to this process. According to the latest combined analysis by the ATLAS and CMS Collaborations on their Higgs measurements [8], the branching fraction of $h$ decay into channels beyond the SM is $\mathcal{B}_{\mathrm{BSM}}^{\exp }=0.00^{+0.16}$, which can be interpreted as placing a cap on the invisible decay of $h$, explicitly $\mathcal{B}(h \rightarrow \text { invisible })_{\exp }<0.16$. Consequently, we can demand

$$
\mathcal{B}(h \rightarrow \bar{\psi} \psi)=\frac{\Gamma(h \rightarrow \bar{\psi} \psi)}{\Gamma_{h}}<0.16 .
$$

In numerical work, we fix $m_{h}=125.1 \mathrm{GeV}$, based on the current data [62], and correspondingly the SM Higgs width $\Gamma_{h}^{\mathrm{SM}}=4.08 \mathrm{MeV}$ [63].

\footnotetext{
${ }^{2}$ Further phenomenology of the DM described in eq. (2.1) has been explored before in [14, 45-54]. Different possibilities for its ultraviolet (UV) completion have also been proposed in [54-61].
} 
Another major constraint on $\lambda_{\psi h}$ is supplied by direct detection experiments, which look for nuclear recoil effects caused by the DM colliding with a nucleon, $N$, nonrelativistically at momentum transfers small relative to the nucleon mass, $m_{N}$. In the $\mathrm{SM}+\psi$, this is an elastic transition, $\psi N \rightarrow \psi N$, which is mediated by the Higgs in the $t$ channel. Its cross section is

$$
\sigma_{\mathrm{el}}^{N}=\frac{\lambda_{\psi h}^{2} g_{N N h}^{2} m_{\psi}^{2} m_{N}^{2}}{\pi\left(m_{\psi}+m_{N}\right)^{2} m_{h}^{4}} .
$$

where $g_{N N h}$ is the effective Higgs-nucleon coupling. Numerically, we adopt $g_{N N h}=0.0011$ following ref. [9]. The strongest restraints on $\sigma_{\mathrm{el}}^{N}$ to date for $m_{\psi} \gtrsim 5 \mathrm{GeV}$ are provided by LUX [1] and PandaX-II [2].

Given that $\Lambda_{\psi}^{-1}=\lambda_{\psi h} / v$ in eq. (2.2) is the coefficient of a dimension-5 effective operator, the size of $\lambda_{\psi h}$ is also capped by the extent of validity of the effective field theory (EFT) description for the $\psi-\mathrm{H}$ interactions. To derive a rough estimate on the minimum of $\Lambda_{\psi}$, we entertain the possibility that this operator is induced by a tree-level diagram mediated by a heavy scalar $X$ with mass $m_{X}$ and couplings to $\psi$ and $h$ described by $\mathcal{L}_{X} \supset-g_{\psi} \bar{\psi} \psi X-g_{h} h^{2} X$ in the UV-complete theory. In addition, we suppose that $g_{\psi} \sim m_{\psi} / v_{X}$ and $g_{h} \sim \lambda_{h X} v_{X}$, where $v_{X}$ is the VEV of $X$ and $\lambda_{h X}$ is dimensionless, inspired by the fermionic and scalar couplings in the SM, ignoring potential modifications due to $h$-X mixing. The EFT will then remain a good approximation and perturbative if $1 /\left|\Lambda_{\psi}\right| \sim 2\left|\lambda_{h X}\right| m_{\psi} / m_{X}^{2}<\left|\lambda_{h X}\right| /\left(2 m_{\psi}\right)<2 \pi / m_{\psi}$, as the $s$-channel $\bar{\psi} \psi$ energy $\sqrt{s}$ satisfies $m_{X}^{2}>s>4 m_{\psi}^{2}$ and $\left|\lambda_{h X}\right|<4 \pi$ for perturbativity. ${ }^{3}$ We then have $\left|\lambda_{\psi h}\right|<2 \pi v / m_{\psi}$. As this follows from the most relaxed requisite on $\lambda_{h X}$, it is likely that the EFT description breaks down at a significantly smaller $\lambda_{\psi h}$. Therefore, alternatively it is reasonable to set $\left|\lambda_{h X}\right|<2$, leading to $\left|\lambda_{\psi h}\right|<v / m_{\psi}$. In the $m_{\psi}<m_{h} / 2$ region, this restriction turns out to be far weaker than that from eq. (2.5) for the Higgs invisible decay, as will be seen shortly.

To illustrate how the model confronts these different requirements, we display in figure 1(a) the values of $\left|\lambda_{\psi h}\right|$ derived from the observed DM relic abundance (green solid curve) and compare them to the upper limits on $\left|\lambda_{\psi h}\right|$ inferred from eq. (2.5) based on LHC data [8] (black dotted curve) and from the validity extent of the EFT approach. For the latter, based on the discussion in the preceding paragraph we draw the magenta band corresponding to the region $\left|\lambda_{\psi h}\right| \in[1,2 \pi] v / m_{\psi}$ in which the EFT description may be expected to have broken down. Thus, we can regard the lower boundary of this band as the upper limit for the reliability of the EFT approximation. We determine the range allowed by these constraints to be $54 \mathrm{GeV} \lesssim m_{\psi} \lesssim 0.8 \mathrm{TeV}$, which translates into the solid portion of the green curve in figure 1(b) for the $\psi$ - $N$ cross-section, $\sigma_{\mathrm{el}}^{N}$. This green solid curve turns out to be forbidden by the LUX bound, except in a slender zone near the Higgs pole, more precisely $55.8 \mathrm{GeV} \lesssim m_{\psi} \lesssim 62.3 \mathrm{GeV}$. Similar results were found in refs. [10, 14]. The ongoing PandaX-II as well as the planned XENON1T, DarkSide G2, and LZ experiments [5] will likely be able to probe the surviving parameter space exhaustively.

\footnotetext{
${ }^{3}$ The same bound on $\Lambda_{\psi}$ was obtained in $[14,64]$ using similar arguments.
} 

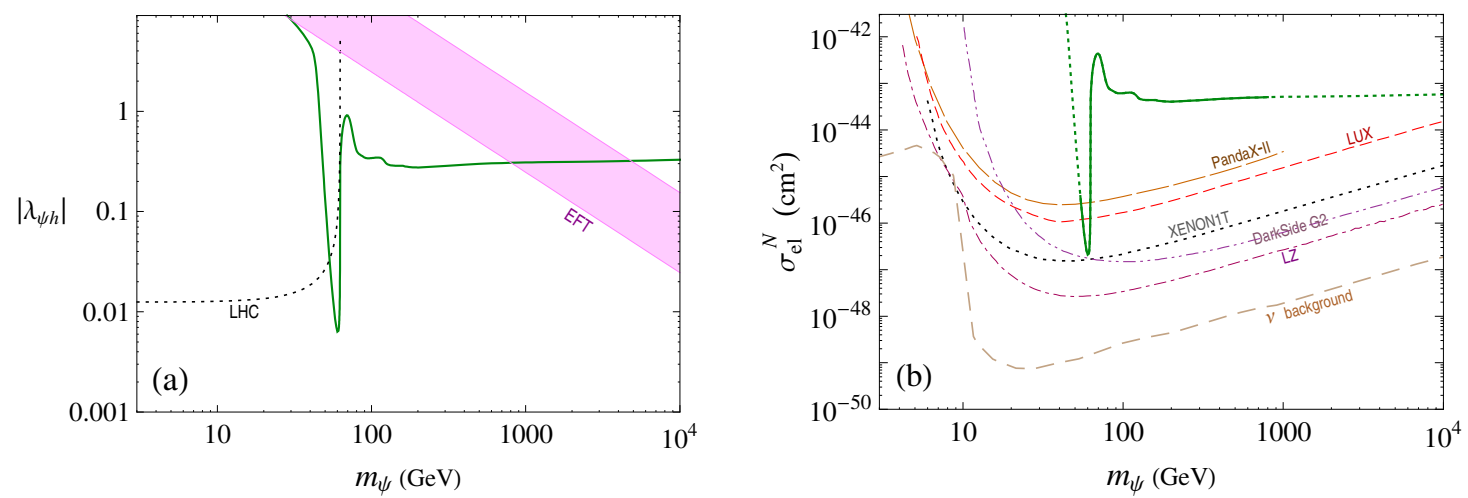

Figure 1. (a) The magnitude of DM-Higgs coupling $\lambda_{\psi h}$ versus DM mass $m_{\psi}$ in the SM+ $\psi$ satisfying the relic density requirement (green curve), compared to the upper limits inferred from LHC data on Higgs invisible decay (black dotted curve) and from the validity extent of the EFT approximation (lower side of magenta band, as discussed in the text). (b) The corresponding crosssection $\sigma_{\mathrm{el}}^{N}$ of $\psi$-nucleon elastic scattering (green curve), compared to the measured upper-limits at 90\% confidence level from LUX [1] (red dashed curve) and PandaX-II [2] (orange long-dashed curve). Also shown are the sensitivity projections [5] of XENON1T [65] (black dotted curve), DarkSide G2 [66] (purple dash-dot-dotted curve), and LZ [67] (maroon dash-dotted curve), and the WIMP discovery lower-limit due to coherent neutrino scattering backgrounds [68] (brown dashed curve). The dotted portions of the green curve are excluded by the LHC and EFT restrictions in (a).

It is worth remarking that the $\lambda_{\psi h}$ values in figure 1(a) are much bigger than most of their counterparts in the simplest scalar-DM model [9-13]. This enlargement is compensation for the suppression of the DM annihilation rate, $\sigma_{\mathrm{ann}} v_{\text {rel }}$, by two powers of the c.m. relative speed $v_{\text {rel }}$ of $\bar{\psi}$ and $\psi$ in the nonrelativistic limit, as can be easily checked. ${ }^{4} \mathrm{As}$ a consequence, the enhanced prediction for $\sigma_{\mathrm{el}}^{N}$ is in conflict with the LUX bound over a wider mass region than in the scalar-DM case.

\subsection{Spin-3/2 dark matter}

The WIMP DM in this scenario is described by a Rarita-Schwinger field [69] which is denoted here by a Dirac four-spinor $\Psi_{\nu}$ with a vector index $\nu$ and satisfies the relation $\gamma^{\nu} \Psi_{\nu}=0 .{ }^{5}$ In the minimal model, called $\mathrm{SM}+\Psi$, the DM is a SM-gauge singlet, its stability is maintained by an unbroken $Z_{2}$ symmetry under which $\Psi_{\nu} \rightarrow-\Psi_{\nu}$, the SM fields being unaffected, and the Higgs-portal interactions arise from dimension-5 operators [51, 52], like in the $\mathrm{SM}+\psi$. The DM Lagrangian, assumed again to be $C P$ invariant, is then

$$
\mathcal{L}_{\Psi}=-\overline{\Psi_{\nu}}\left(i \not \partial-\mu_{\Psi}\right) \Psi^{\nu}+\frac{\overline{\Psi_{\nu}} \Psi^{\nu} \mathrm{H}^{\dagger} \mathrm{H}}{\Lambda_{\Psi}} \supset m_{\Psi} \overline{\Psi_{\nu}} \Psi^{\nu}+\lambda_{\Psi h} \overline{\Psi_{\nu}} \Psi^{\nu}\left(h+\frac{h^{2}}{2 v}\right)
$$

\footnotetext{
${ }^{4}$ If $C P$ invariance is not imposed on $\mathcal{L}_{\psi}$ in eq. (2.1), it can accommodate the combination $\bar{\psi} \gamma_{5} \psi \mathbf{H}^{\dagger} \mathrm{H}$. After electroweak symmetry breaking, this operator generally gives rise to both scalar and pseudoscalar contributions to DM annihilation, $h \rightarrow \bar{\psi} \psi$, and DM-nucleon scattering [14,53]. The pseudoscalar one can alleviate the $v_{\text {rel }}^{2}$ suppression in $\sigma_{\text {ann }} v_{\text {rel }}$, but yields a tiny effect on the DM-nucleon cross-section.

${ }^{5}$ The basic properties of this kind of spin- $3 / 2$ fermion, especially in the DM context, have been elaborated in $[51,52,70-76]$.
} 
where $\mu_{\Psi}$ and $\Lambda_{\Psi}$ are real constants, $\Lambda_{\Psi}$ encodes the underlying heavy physics, and the DM mass and coupling

$$
m_{\Psi}=\mu_{\Psi}+\frac{\lambda_{\Psi h} v}{2}, \quad \lambda_{\Psi h}=\frac{v}{\Lambda_{\Psi}}
$$

respectively, are the only free parameters in $\mathcal{L}_{\Psi}$.

Accordingly, we can derive the main quantities relevant to the DM phenomenology in analogy to the spin- $1 / 2$ case. Thus, the DM-annihilation cross-section $\sigma_{\text {ann }}$ is given by ${ }^{6}$

$$
\begin{gathered}
\sigma_{\mathrm{ann}}=\sigma\left(\bar{\Psi} \Psi \rightarrow h^{*} \rightarrow X_{\mathrm{SM}}\right)+\sigma(\bar{\Psi} \Psi \rightarrow h h), \\
\sigma\left(\bar{\Psi} \Psi \rightarrow h^{*} \rightarrow X_{\mathrm{SM}}\right)=\frac{\left(5 \beta_{\Psi}-6 \beta_{\Psi}^{3}+9 \beta_{\Psi}^{5}\right) \lambda_{\Psi h}^{2} s^{5 / 2} \sum_{i} \Gamma\left(\tilde{h} \rightarrow X_{i, \mathrm{SM}}\right)}{576 m_{\Psi}^{4}\left[\left(m_{h}^{2}-s\right)^{2}+\Gamma_{h}^{2} m_{h}^{2}\right]}, \quad X_{\mathrm{SM}} \neq h h,
\end{gathered}
$$

where $\beta_{\mathrm{X}}$ is defined in eq. (2.3), the formula for $\sigma(\bar{\Psi} \Psi \rightarrow h h)$ is described in appendix A, and the Higgs' width $\Gamma_{h}=\Gamma_{h}^{\mathrm{SM}}+\Gamma(h \rightarrow \bar{\Psi} \Psi)$ receives a contribution from the invisible channel $h \rightarrow \bar{\Psi} \Psi$ if $2 m_{\Psi}<m_{h}$. From eq. (2.7), we derive

$$
\Gamma(h \rightarrow \bar{\Psi} \Psi)=\frac{\lambda_{\Psi h}^{2} m_{h}}{8 \pi} \frac{\left(1-6 R_{\Psi}^{2}+18 R_{\Psi}^{4}\right)}{9 R_{\Psi}^{4}}\left(1-4 R_{\Psi}^{2}\right)^{3 / 2}, \quad R_{\Psi}=\frac{m_{\Psi}}{m_{h}},
$$

which is subject to

$$
\mathcal{B}(h \rightarrow \bar{\Psi} \Psi)=\frac{\Gamma(h \rightarrow \bar{\Psi} \Psi)}{\Gamma_{h}}<0.16
$$

based on the aforementioned LHC Higgs data [8]. For the $h$-mediated scattering $\Psi N \rightarrow$ $\Psi N$, the cross section is

$$
\sigma_{\mathrm{el}}^{N}=\frac{\lambda_{\Psi h}^{2} g_{N N h}^{2} m_{\Psi}^{2} m_{N}^{2}}{\pi\left(m_{\Psi}+m_{N}\right)^{2} m_{h}^{4}} .
$$

With the formulas above, we arrive at the $\left|\lambda_{\Psi h}\right|$ values consistent with the observed relic density and the corresponding $\sigma_{\mathrm{el}}^{N}$, which are depicted by the green curves in figures 2(a) and 2(b), respectively. In figure 2(a), the black dotted curve marks the upper bound on $\left|\lambda_{\Psi h}\right|$ from the Higgs measurements, and the magenta band represents the zone $\left|\lambda_{\Psi h}\right| \in[1,2 \pi] v / m_{\Psi}$ in which the EFT description may be expected to have broken down, analogously to the spin- $1 / 2$ case. Consequently, like before the lower boundary of this band may be taken to be the upper limit for the validity of the EFT approximation. The $m_{\Psi}$ range fulfilling these requirements translates into the solid part of the green curve in figure 2(b) for the $\Psi$-nucleon cross-section, $\sigma_{\mathrm{el}}^{N}$. Evidently, the direct search bounds reduce the viable DM-mass region in the $\mathrm{SM}+\Psi$ relative to the $\mathrm{SM}+\psi$. More precisely, only $58.0 \mathrm{GeV} \lesssim m_{\Psi} \lesssim 61.8 \mathrm{GeV}$ remains viable. This minuscule stretch toward the bottom of the Higgs-resonance dip is almost eliminated by the final LUX limit and will likely be fully excluded by the forthcoming probes of PandaX-II, unless it discovers $\Psi$.

\footnotetext{
${ }^{6}$ The $\bar{\Psi} \Psi$ annihilation rate, $\sigma_{\text {ann }} v_{\text {rel }}$, like its spin- $1 / 2$ counterpart, suffers from $v_{\text {rel }}^{2}$ suppression in the nonrelativistic limit. If $\mathcal{L}_{\Psi}$ is not $C P$-invariant, it can include the pseudoscalar combination $\overline{\Psi_{\nu}} \gamma_{5} \Psi^{\nu} \mathrm{H}^{\dagger} \mathrm{H}$. In its presence, there is generally an admixture of scalar and pseudoscalar contributions to $\Psi$ - $h$ interactions which can ameliorate the $v_{\text {rel }}^{2}$ suppression in $\sigma_{\text {ann }} v_{\text {rel }}$.
} 

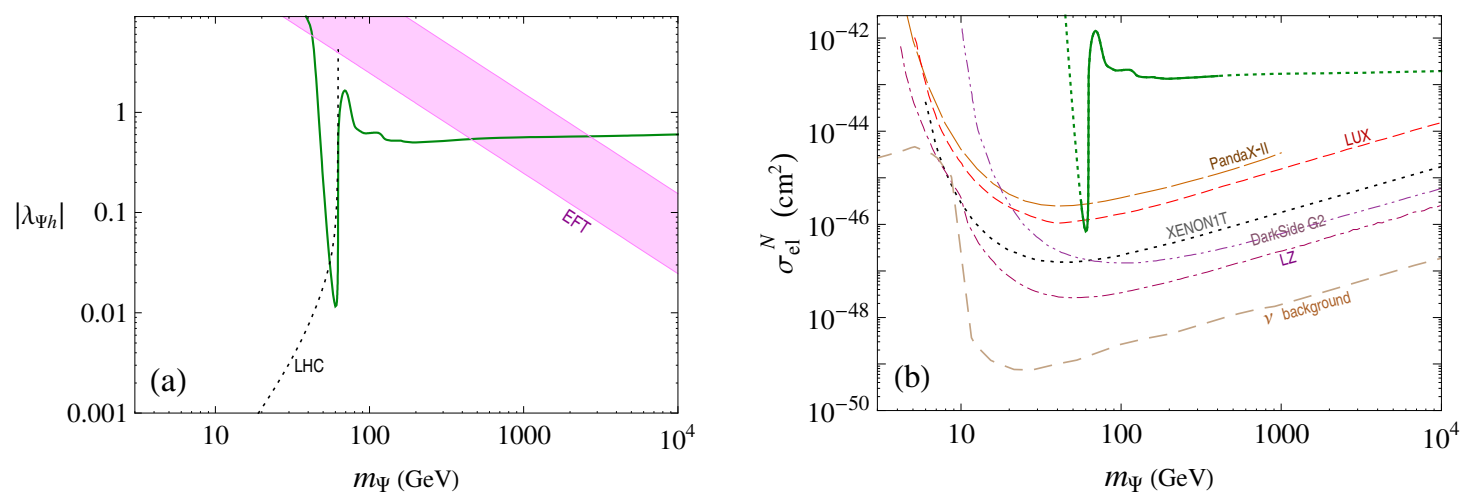

Figure 2. The same as figure 1, except the DM is the spin-3/2 singlet $\Psi$ in the $\mathrm{SM}+\Psi$.

\subsection{Vector dark matter}

In the minimal model, dubbed $\mathrm{SM}+\mathrm{V}$, the only new ingredient beyond the $\mathrm{SM}$ is a spin-1 state acting as the WIMP DM candidate [48]. We assume that it is described by a real field $V_{\nu}$ which is a singlet under the SM gauge group and odd under an unbroken $Z_{2}$ symmetry which does not alter SM members. Unlike its fermionic counterparts, $V_{\nu}$ can couple to the Higgs doublet via a dimension-four operator. The DM Lagrangian that respects the SM gauge symmetry is then [48]

$$
\mathcal{L}_{V}=-\frac{1}{4} V_{\kappa \nu} V^{\kappa \nu}+\frac{\mu_{V}^{2}}{2} V_{\kappa} V^{\kappa}+\frac{\lambda_{V}}{4}\left(V_{\kappa} V^{\kappa}\right)^{2}+\lambda_{h} \mathrm{H}^{\dagger} \mathrm{H} V_{\kappa} V^{\kappa},
$$

where $V_{\kappa \nu}=\partial_{\kappa} V_{\nu}-\partial_{\nu} V_{\kappa}$ and $\mu_{V}, \lambda_{V}$, and $\lambda_{h}$ are real constants. Although the terms in $\mathcal{L}_{V}$ are at most of dimension four, it is actually nonrenormalizable and violates unitarity [77]. ${ }^{7}$ Hereafter, we make no assumption about the details of the UV completion of $\mathcal{L}_{V}$, implying that $V$ is not necessarily a gauge boson and may even be a composite object in the dark sector. Consequently, we can generally treat $\mu_{V}$ and $\lambda_{V, h}$ as independent parameters. For the calculations below, $\lambda_{V}$ does not play any essential role, and so we can express

$$
\mathcal{L}_{V} \supset \frac{m_{V}^{2}}{2} V_{\kappa} V^{\kappa}+\lambda_{h}\left(h v+\frac{h^{2}}{2}\right) V_{\kappa} V^{\kappa}
$$

where $m_{V}=\left(\mu_{V}^{2}+\lambda_{h} v^{2}\right)^{1 / 2}$ is the $V$ mass.

As in the fermionic DM models, $\lambda_{h}$ has to meet the different requirements on DM annihilation, invisible decay $h \rightarrow V V$, and DM-nucleon scattering. From eq. (2.14), we obtain

$$
\begin{gathered}
\sigma_{\mathrm{ann}}=\sigma\left(\mathrm{VV} \rightarrow h^{*} \rightarrow X_{\mathrm{SM}}\right)+\sigma(\mathrm{VV} \rightarrow h h), \\
\sigma\left(\mathrm{VV} \rightarrow h^{*} \rightarrow X_{\mathrm{SM}}\right)=\frac{\lambda_{h}^{2}\left(\beta_{V}^{2} s^{2}+12 m_{V}^{4}\right) v^{2} \sum_{i} \Gamma\left(\tilde{h} \rightarrow X_{i, \mathrm{SM}}\right)}{9 \beta_{V} m_{V}^{4} \sqrt{s}\left[\left(m_{h}^{2}-s\right)^{2}+\Gamma_{h}^{2} m_{h}^{2}\right]}, \quad X_{\mathrm{SM}} \neq h h,
\end{gathered}
$$

\footnotetext{
${ }^{7}$ This type of Higgs-portal vector-DM model has been explored previously in [10, 14, 48-52]. Examples of its UV completion were proposed in [61, 78-80].
} 
the formula for $\sigma(\mathrm{VV} \rightarrow h h)$ is relegated to appendix A and the $h$ width $\Gamma_{h}=\Gamma_{h}^{\mathrm{SM}}+\Gamma(h \rightarrow$ VV), with

$$
\Gamma(h \rightarrow V V)=\frac{\lambda_{h}^{2} v^{2}}{8 \pi m_{h}} \frac{\left(1-4 R_{V}^{2}+12 R_{V}^{4}\right)}{4 R_{V}^{4}} \sqrt{1-4 R_{V}^{2}}, \quad R_{V}=\frac{m_{V}}{m_{h}} .
$$

We will again demand

$$
\mathcal{B}(h \rightarrow V V)=\frac{\Gamma(h \rightarrow V V)}{\Gamma_{h}}<0.16
$$

based on the Higgs data [8]. For the Higgs-mediated DM-nucleon collision $V N \rightarrow V N$, the cross section is

$$
\sigma_{\mathrm{el}}^{N}=\frac{\lambda_{h}^{2} g_{N N h}^{2} m_{N}^{2} v^{2}}{\pi\left(m_{V}+m_{N}\right)^{2} m_{h}^{4}} .
$$

There are also theoretical considerations relevant to restraining $\lambda_{h}$. Since $\mathcal{L}_{V}$ in eq. (2.14) leads to unitarity violation [77], we need to ensure that it does not occur with the extracted $\lambda_{h}$ values. As discussed in appendix B, this implies that we need to have $\left|\lambda_{h}\right|<\sqrt{2 \pi} m_{V} / v$. There is additionally a complementary restraint from the expectation that the theory remains perturbative. As also explained in appendix $\mathrm{B}$, this translates into the bound $\left|\lambda_{h}\right|<1$.

In figure 3(a), we present the $\lambda_{h}$ values fulfilling the relic density requirement (green curve). Also shown are the upper limits from the Higgs invisible decay data (black dashed curve) and from the unitarity and perturbativity considerations (maroon dashed curves). We plot the corresponding $V$-nucleon cross-section from eq. (2.18) in figure $3(\mathrm{~b})$, where the dotted sections of the green curve are disallowed by the restrictions in figure $3(\mathrm{a})$. We find that $m_{V}$ values approximately below $54 \mathrm{GeV}$ and from $62.6 \mathrm{GeV}$ to $1.42 \mathrm{TeV}$ are in conflict with LHC and LUX data. Comparable results were obtained in ref. [10]. However, the graphs also reveal that for $m_{V}>3.9 \mathrm{TeV}$ the effective theory probably is no longer perturbative. Thus, overall the situation is rather similar to that in the simplest scalarDM model [9-13], where the annihilation rate does not suffer from the $v_{\text {rel }}^{2}$ suppression and consequently the viable parameter space is far greater than in its fermionic counterparts.

\section{Two-Higgs-doublet-portal fermionic and vector DM models}

Here we explore extensions of the minimal models in the last section by adding in each case another Higgs doublet. Furthermore, we suppose that the SM fermions in the extended scenarios have the Yukawa interactions of the two-Higgs-doublet model (THDM) of type II, where the down-type fermions get mass from only one of the Higgs doublets, $H_{1}$, and the up-type fermions from the other doublet, $H_{2}$. Accordingly, the Yukawa Lagrangian is $[81,82]$

$$
\mathcal{L}_{\mathrm{Y}}=-\bar{Q}_{j, L}\left(\lambda_{2}^{u}\right)_{j l} \tilde{H}_{2} \mathcal{U}_{l, R}-\bar{Q}_{j, L}\left(\lambda_{1}^{d}\right)_{j l} H_{1} \mathcal{D}_{l, R}-\bar{L}_{j, L}\left(\lambda_{1}^{\ell}\right)_{j l} H_{1} E_{l, R}+\text { H.c. },
$$



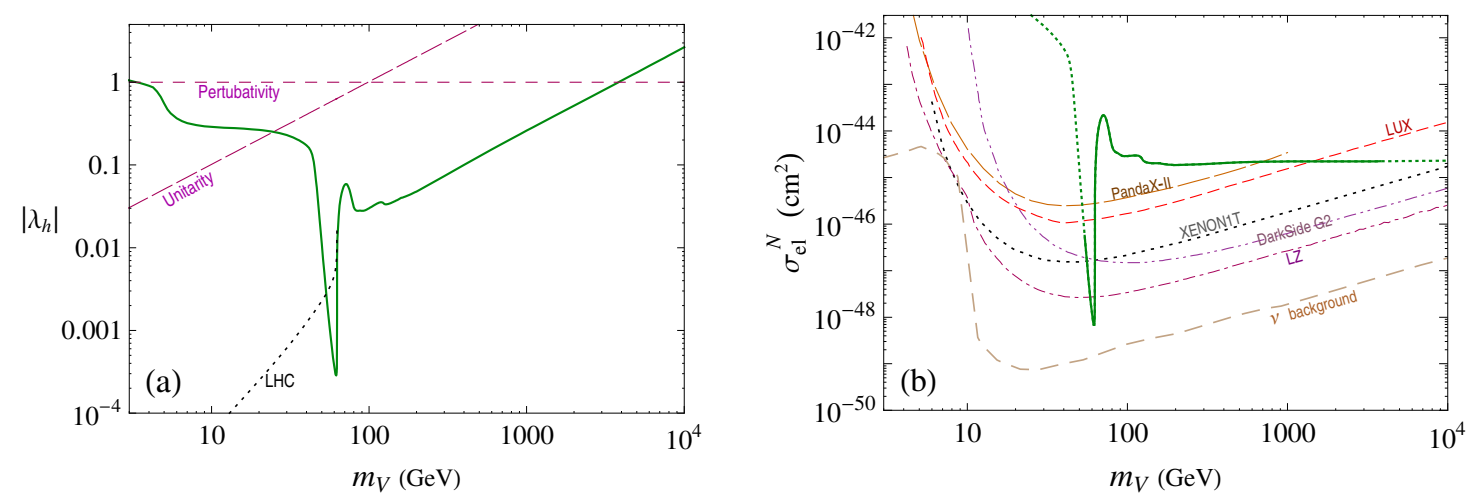

Figure 3. The magnitude of Higgs coupling $\lambda_{h}$ to the vector DM versus its mass in the SM+V satisfying the relic abundance requirement (green curve), compared to the upper limits inferred from Higgs invisible decay data (black dotted curve) and from unitarity and perturbativity considerations (maroon dashed curves). (b) The corresponding cross-section $\sigma_{\mathrm{el}}^{N}$ of DM-nucleon scattering (green curve) compared to the same data and projections as in figure 1(b). The dotted sections of the green curve are disallowed by the constraints in (a).

where $j, l=1,2,3$ are summed over, $Q_{j, L}\left(L_{j, L}\right)$ stands for left-handed quark (lepton) doublets, $\mathcal{U}_{l, R}$ and $\mathcal{D}_{l, R}\left(E_{l, R}\right)$ are right-handed quark (charged lepton) fields, $\tilde{H}_{1,2}=$ $i \tau_{2} H_{1,2}^{*}$ with $\tau_{2}$ being the second Pauli matrix, and $\lambda^{u, d, \ell}$ represent $3 \times 3$ Yukawa-coupling matrices. This Lagrangian respects another discrete symmetry, $Z_{2}^{\prime}$, under which $H_{2} \rightarrow$ $-H_{2}$ and $\mathcal{U}_{R} \rightarrow-\mathcal{U}_{R}$, while all the other fields are not affected. Thus, $Z_{2}^{\prime}$ prohibits the combinations $\bar{Q}_{L} \tilde{H}_{1} \mathcal{U}_{R}, \bar{Q}_{L} H_{2} \mathcal{D}_{R}, \bar{L}_{L} H_{2} E_{R}$, and their Hermitian conjugates from entering $\mathcal{L}_{\mathrm{Y}}$.

In the scalar sector, the renormalizable potential $\mathcal{V}_{H}$ is that of the THDM II,

$$
\begin{aligned}
\mathcal{V}_{H}= & m_{11}^{2} H_{1}^{\dagger} H_{1}+m_{22}^{2} H_{2}^{\dagger} H_{2}-\left(m_{12}^{2} H_{1}^{\dagger} H_{2}+\text { H.c. }\right)+\frac{\lambda_{1}}{2}\left(H_{1}^{\dagger} H_{1}\right)^{2}+\frac{\lambda_{2}}{2}\left(H_{2}^{\dagger} H_{2}\right)^{2} \\
& +\lambda_{3} H_{1}^{\dagger} H_{1} H_{2}^{\dagger} H_{2}+\lambda_{4} H_{1}^{\dagger} H_{2} H_{2}^{\dagger} H_{1}+\frac{\lambda_{5}}{2}\left[\left(H_{1}^{\dagger} H_{2}\right)^{2}+\text { H.c. }\right] .
\end{aligned}
$$

Although dimension-4 combinations with an odd number of $H_{2}^{(\dagger)}$ cannot appear due to $Z_{2}^{\prime}$, in $\mathcal{V}_{H}$ we have allowed the $m_{12}^{2}$ terms which softly break $Z_{2}^{\prime}$ and are important in relaxing the caps on the Higgs masses [82]. The Hermiticity of $\mathcal{V}_{H}$ implies that $m_{11,22}^{2}$ and $\lambda_{1,2,3,4}$ are real parameters. With $\mathcal{V}_{H}$ chosen to be $C P$ invariant, $m_{12}^{2}$ and $\lambda_{5}$ are also real constants.

To see how $\mathcal{V}_{H}$ describes the couplings among the physical states in the Higgs doublets, we first decompose them as

$$
H_{r}=\frac{1}{\sqrt{2}}\left(\begin{array}{c}
\sqrt{2} h_{r}^{+} \\
v_{r}+h_{r}^{0}+i I_{r}^{0}
\end{array}\right), \quad r=1,2,
$$

where $v_{1,2}$ denote the VEVs of $H_{1,2}$, respectively, and are linked to the electroweak mass scale $v \simeq 246 \mathrm{GeV}$ by $v_{1}=v \cos \beta$ and $v_{2}=v \sin \beta$. The components $h_{r}^{+}, h_{r}^{0}$, and $I_{r}^{0}$ are 
connected to the physical Higgs bosons $h, H, A$, and $H^{+}$by

$$
\begin{array}{rlr}
\left(\begin{array}{l}
h_{1}^{+} \\
h_{2}^{+}
\end{array}\right) & =\left(\begin{array}{rr}
c_{\beta} & -s_{\beta} \\
s_{\beta} & c_{\beta}
\end{array}\right)\left(\begin{array}{l}
w^{+} \\
H^{+}
\end{array}\right), \quad\left(\begin{array}{l}
I_{1}^{0} \\
I_{2}^{0}
\end{array}\right)=\left(\begin{array}{rr}
c_{\beta} & -s_{\beta} \\
s_{\beta} & c_{\beta}
\end{array}\right)\left(\begin{array}{l}
z \\
A
\end{array}\right), \\
\left(\begin{array}{l}
h_{1}^{0} \\
h_{2}^{0}
\end{array}\right) & =\left(\begin{array}{rr}
c_{\alpha} & -s_{\alpha} \\
s_{\alpha} & c_{\alpha}
\end{array}\right)\left(\begin{array}{c}
H \\
h
\end{array}\right), & c_{\mathcal{X}}=\cos \mathcal{X}, \quad s_{\mathcal{X}}=\sin \mathcal{X},
\end{array}
$$

where $\mathcal{X}$ is any angle or combination of angles and $w^{ \pm}$and $z$ are, respectively, the would-be Goldstone bosons that will be eaten by the $W^{ \pm}$and $Z$ bosons. We can then express the terms in $\mathcal{V}_{H}$ after electroweak symmetry breaking that are relevant to our purposes as

$$
\begin{aligned}
\mathcal{V}_{H} \supset & \left(\frac{1}{6} \lambda_{h h h} h^{2}+\frac{1}{2} \lambda_{h h H} h H+\frac{1}{2} \lambda_{h H H} H^{2}+\frac{1}{2} \lambda_{h A A} A^{2}+\lambda_{h H^{+} H^{-}} H^{+} H^{-}\right) h v \\
& +\left(\frac{1}{6} \lambda_{H H H} H^{2}+\frac{1}{2} \lambda_{H A A} A^{2}+\lambda_{H H^{+} H^{-}} H^{+} H^{-}\right) H v
\end{aligned}
$$

where the $\lambda_{\mathrm{s}}$ are linked to the physical Higgs masses [82] and the relations are listed in ref. [9].

Since $h$ and $H$ couple directly to the $W$ and $Z$ bosons, we need to take into account $\mathrm{DM}$ annihilation into $W^{+} W^{-}$and $Z Z$. The pertinent interactions are given by

$$
\mathcal{L} \supset\left(2 m_{W}^{2} W^{+\nu} W_{\nu}^{-}+m_{Z}^{2} Z^{\nu} Z_{\nu}\right)\left(k_{V}^{h} \frac{h}{v}+k_{V}^{H} \frac{H}{v}\right), \quad k_{V}^{h}=s_{\beta-\alpha}, \quad k_{V}^{H}=c_{\beta-\alpha} .
$$

The presence of the extra Higgs doublet not only offers another portal between the dark and visible sectors, but also can produce modifications to the effective coupling between a Higgs boson $\mathcal{H}$ and a nucleon $\mathcal{N}$ which is defined by

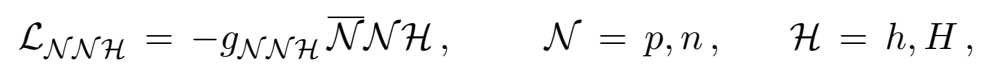

and plays a crucial role in DM-nucleon collisions. The potential changes spring from the quark-Higgs terms in eq. (3.1)

$$
\mathcal{L}_{\mathrm{Y}} \supset-\sum_{q} k_{q}^{\mathcal{H}} m_{q} \bar{q} q \frac{\mathcal{H}}{v}, \quad k_{c, t}^{\mathcal{H}}=k_{u}^{\mathcal{H}}, \quad k_{s, b}^{\mathcal{H}}=k_{d}^{\mathcal{H}},
$$

where the sum is over all quarks, $q=u, d, s, c, b, t$, and

$$
k_{u}^{h}=\frac{c_{\alpha}}{s_{\beta}}, \quad k_{d}^{h}=-\frac{s_{\alpha}}{c_{\beta}}, \quad k_{u}^{H}=\frac{s_{\alpha}}{s_{\beta}}, \quad k_{d}^{H}=\frac{c_{\alpha}}{c_{\beta}},
$$

which are generally different from the SM values $k_{q}^{h}=1$ and $k_{q}^{H}=0$. Relating the above quark- and hadronic-level quantities, one arrives numerically at $[9,15,20]$

$$
g_{p p \mathcal{H}}=\left(0.5631 k_{u}^{\mathcal{H}}+0.5599 k_{d}^{\mathcal{H}}\right) \times 10^{-3}, \quad g_{n n \mathcal{H}}=\left(0.5481 k_{u}^{\mathcal{H}}+0.5857 k_{d}^{\mathcal{H}}\right) \times 10^{-3} .
$$

Setting $k_{u, d}^{h}=1$ in the $\mathcal{H}=h$ formulas, we reproduce the SM values $g_{p p h, n n h}^{\mathrm{SM}} \simeq 0.0011$ quoted in the last section. However, if $k_{u, d}^{\mathcal{H}}$ are away from their SM expectations, $g_{p p \mathcal{H}}$ 
and $g_{n n \mathcal{H}}$ can be very dissimilar, manifesting considerable isospin-violation. Especially, if $k_{u, d}^{\mathcal{H}}$ have opposite signs, it may be possible to reduce $g_{\mathcal{N N \mathcal { H }}}$ such that the DM effective interactions with nucleons become weak enough to evade the experimental constraints.

This suggests that to address DM-nucleon collisions in models with a THDM-II portal it is more appropriate to work with either the DM-proton or -neutron cross-section $\left(\sigma_{\mathrm{el}}^{p}\right.$ or $\sigma_{\mathrm{el}}^{n}$, respectively) rather than the DM-nucleon one $\left(\sigma_{\mathrm{el}}^{N}\right)$ under the assumption of isospin conservation. For comparison with experiment, in case the DM effective interactions with nucleons violate isospin, the computed $\sigma_{\mathrm{el}}^{p, n}$ can be converted to $\sigma_{\mathrm{el}}^{N}$ by means of [38-41]

$$
\sigma_{\mathrm{el}}^{N} \sum_{i} \eta_{i} \mu_{A_{i}}^{2} A_{i}^{2}=\sigma_{\mathrm{el}}^{p} \sum_{i} \eta_{i} \mu_{A_{i}}^{2}\left[\mathcal{Z}+\left(A_{i}-\mathcal{Z}\right) f_{n} / f_{p}\right]^{2}, \quad \sigma_{\mathrm{el}}^{n}=\sigma_{\mathrm{el}}^{p} f_{n}^{2} / f_{p}^{2},
$$

where each sum is over isotopes of the element in the target material with which the DM interacts dominantly, $\eta_{i}\left(A_{i}\right)$ stand for the fractional abundances (the nucleon numbers) of the isotopes, $\mu_{A_{i}}=m_{A_{i}} m_{\psi} /\left(m_{A_{i}}+m_{\psi}\right)$, with $m_{A_{i}}$ being the $i$ th isotope's mass, $\mathcal{Z}$ represents the proton number of the element, and $f_{p(n)}$ is the effective coupling of the DM to the proton (neutron).

In what follows, we select $h$ to be the $125-\mathrm{GeV}$ Higgs boson. Accordingly, $k_{d, u, V}^{h}$ in eq. (3.9) need to be compatible with LHC measurements on the $h$ couplings to SM fermions and electroweak bosons. The modification to the $h \rightarrow X \bar{X}$ interaction due to new physics can be parameterized by $\kappa_{X}$ in $\kappa_{X}^{2}=\Gamma_{h \rightarrow X \bar{X}} / \Gamma_{h \rightarrow X \bar{X}}^{\mathrm{SM}}$. Assuming that $\left|\kappa_{W, Z}\right| \leq 1$ and the total width of $h$ can be altered by decay modes beyond the SM, the ATLAS and CMS Collaborations have carried out simultaneous fits to their Higgs data to extract [8]

$$
\begin{aligned}
& \kappa_{W}=0.90 \pm 0.09, \quad \kappa_{t}=1.43_{-0.22}^{+0.23}, \quad\left|\kappa_{b}\right|=0.57 \pm 0.16, \quad\left|\kappa_{\gamma}\right|=0.90_{-0.09}^{+0.10}, \\
& \kappa_{Z}=1.00_{-0.08}, \quad\left|\kappa_{g}\right|=0.81_{-0.10}^{+0.13}, \quad\left|\kappa_{\tau}\right|=0.87_{-0.11}^{+0.12},
\end{aligned}
$$

where [8] $\kappa_{\gamma}^{2}=0.07 \kappa_{t}^{2}+1.59 \kappa_{W}^{2}-0.66 \kappa_{t} \kappa_{W}$. In the context of the THDM II, we expect these numbers to obey the relations $k_{V}^{h}=\kappa_{W}=\kappa_{Z}, k_{u}^{h}=\kappa_{t} \simeq \kappa_{g}$, and $k_{d}^{h}=\kappa_{b}=\kappa_{\tau}$ within one sigma, but the $\kappa_{t, g}\left(\kappa_{b, \tau}\right)$ numbers above overlap only at the two-sigma level. Pending improvement in the precision of these parameters from future data and following ref. [9], based on eq. (3.12) we can then impose

$$
0.81 \leq k_{V}^{h} \leq 1, \quad 0.71 \leq k_{u}^{h} \leq 1.66, \quad 0.41 \leq\left|k_{d}^{h}\right| \leq 0.99, \quad 0.81 \leq\left|k_{\gamma}^{h}\right| \leq 1,
$$

where $k_{\gamma}^{h}$ includes the loop contribution of $H^{ \pm}$to $h \rightarrow \gamma \gamma$, and so $k_{\gamma}^{h} \rightarrow \kappa_{\gamma}$ if the impact of $H^{ \pm}$is vanishing.

There are other constraints that we need to consider as well. The extra Higgs particles in the THDM generally modify the so-called oblique electroweak parameters $S$ and $T$ encoding the impact of new physics coupled to the standard $\mathrm{SU}(2)_{L}$ gauge boson [83], and so the new scalars must also conform to the empirical requisites on these quantities. To ensure this, we use the pertinent results of ref. [84] and the $S$ and $T$ data from ref. [85].

Theoretically, the parameters of the scalar potential $\mathcal{V}_{H}$ in eq. (3.2) need to fulfill a number of conditions. To keep the theory perturbative, each of the quartic couplings in $\mathcal{V}_{H}$ cannot be too big. Another requirement is the stability of $\mathcal{V}_{H}$, implying that it has to 
be bounded from below. It is also essential to check that the (tree level) amplitudes for scalar-scalar scattering do not violate unitarity constraints. We summarize the expressions pertaining to these conditions in appendix B.

In the rest of this section, we treat in turn the THDM II $+\psi$, THDM II $+\Psi$, and THDM II $+\mathrm{V}$, which are respectively the type-II two-Higgs-doublet extensions of the minimal models of the previous section. Thus, the DM stability is maintained in each case by the exactly conserved $Z_{2}$ symmetry as before, under which the DM is odd and all the other fields are even. In addition, we demand that, besides the scalar potential, the DM sector be $C P$ invariant. We will demonstrate that in the presence of the second doublet it is possible to have substantial weakening of the constraints from DM direct detection experiments or perhaps even to evade them in the future.

\subsection{THDM II $+\psi$}

In this scenario, the Lagrangian for the DM is $[32,33]$

$$
\mathcal{L}_{\psi}^{\prime}=\bar{\psi} i \not \partial \psi-\mu_{\psi} \bar{\psi} \psi-\bar{\psi} \psi\left(\frac{H_{1}^{\dagger} H_{1}}{\Lambda_{1 \psi}}+\frac{H_{2}^{\dagger} H_{2}}{\Lambda_{2 \psi}}\right),
$$

where $\mu_{\psi}$ and $\Lambda_{1 \psi, 2 \psi}$ are real constants of dimension mass and $\Lambda_{1 \psi, 2 \psi}$ contain the parameters of the underlying heavy new physics. The $Z_{2}^{\prime}$ symmetry prevents the combinations $\bar{\psi} \psi H_{1}^{\dagger} H_{2}$ and $\bar{\psi} \psi H_{2}^{\dagger} H_{1}$ from appearing in $\mathcal{L}_{\psi}^{\prime}$.

After electroweak symmetry breaking, we can express the relevant terms in $\mathcal{L}_{\psi}^{\prime}$ involving the physical bosons as

$$
\begin{aligned}
\mathcal{L}_{\psi}^{\prime} \supset & -m_{\psi} \bar{\psi} \psi-\bar{\psi} \psi\left(\lambda_{\psi h} h+\lambda_{\psi H} H\right) \\
& -\frac{\bar{\psi} \psi}{2 v}\left(\lambda_{\psi h h} h^{2}+2 \lambda_{\psi h H} h H+\lambda_{\psi H H} H^{2}+\lambda_{\psi A A} A^{2}+2 \lambda_{\psi H^{+} H^{-}} H^{+} H^{-}\right),
\end{aligned}
$$

where

$$
\begin{aligned}
m_{\psi} & =\mu_{\psi}+\left(\lambda_{1 \psi} c_{\beta}^{2}+\lambda_{2 \psi} s_{\beta}^{2}\right) \frac{v}{2}, & \lambda_{r \psi} & =\frac{v}{\Lambda_{r \psi}}, \quad r=1,2, \\
\lambda_{\psi h} & =\lambda_{2 \psi} c_{\alpha} s_{\beta}-\lambda_{1 \psi} s_{\alpha} c_{\beta}, & \lambda_{\psi H} & =\lambda_{1 \psi} c_{\alpha} c_{\beta}+\lambda_{2 \psi} s_{\alpha} s_{\beta}, \\
\lambda_{\psi h h} & =\lambda_{1 \psi} s_{\alpha}^{2}+\lambda_{2 \psi} c_{\alpha}^{2}, & \lambda_{\psi H H} & =\lambda_{1 \psi} c_{\alpha}^{2}+\lambda_{2 \psi} s_{\alpha}^{2}, \\
\lambda_{\psi h H} & =\left(\lambda_{2 \psi}-\lambda_{1 \psi}\right) c_{\alpha} s_{\alpha}, & \lambda_{\psi A A} & =\lambda_{\psi H^{+} H^{-}}=\lambda_{1 \psi} s_{\beta}^{2}+\lambda_{2 \psi} c_{\beta}^{2} .
\end{aligned}
$$

There is no $\bar{\psi} \psi A$ term under the assumed $C P$ conservation. Since $\mu_{\psi}$ and $\Lambda_{1 \psi, 2 \psi}$ are free parameters, so are $m_{\psi}$ and $\lambda_{\psi h, \psi H}$. The couplings of $\bar{\psi} \psi$ to a pair of Higgs bosons can then be expressed in terms of $\lambda_{\psi h, \psi H}$ as

$$
\begin{aligned}
\lambda_{\psi h h} & =\left(\frac{c_{\alpha}^{3}}{s_{\beta}}-\frac{s_{\alpha}^{3}}{c_{\beta}}\right) \lambda_{\psi h}+\frac{s_{2 \alpha} c_{\beta-\alpha}}{s_{2 \beta}} \lambda_{\psi H}, \quad \lambda_{\psi h H}=\frac{s_{2 \alpha}}{s_{2 \beta}}\left(\lambda_{\psi h} c_{\beta-\alpha}-\lambda_{\psi H} s_{\beta-\alpha}\right), \\
\lambda_{\psi H H} & =\left(\frac{c_{\alpha}^{3}}{c_{\beta}}+\frac{s_{\alpha}^{3}}{s_{\beta}}\right) \lambda_{\psi H}-\frac{s_{2 \alpha} s_{\beta-\alpha}}{s_{2 \beta}} \lambda_{\psi h}, \\
\lambda_{\psi A A} & =\lambda_{\psi H^{+} H^{-}}=\frac{c_{\alpha} c_{\beta}^{3}-s_{\alpha} s_{\beta}^{3}}{c_{\beta} s_{\beta}} \lambda_{\psi h}+\frac{c_{\alpha} s_{\beta}^{3}+s_{\alpha} c_{\beta}^{3}}{c_{\beta} s_{\beta}} \lambda_{\psi H} .
\end{aligned}
$$


If both the $h$ and $H$ couplings to $\psi$ are nonzero, the DM-nucleon scattering $\psi \mathcal{N} \rightarrow \psi \mathcal{N}$ proceeds via tree-level diagrams mediated by $h$ and $H$, leading to the cross section

$$
\sigma_{\mathrm{el}}^{\mathcal{N}}=\frac{m_{\psi}^{2} m_{\mathcal{N}}^{2}}{\pi\left(m_{\psi}+m_{\mathcal{N}}\right)^{2}}\left(\frac{\lambda_{\psi h} g_{\mathcal{N N} h}}{m_{h}^{2}}+\frac{\lambda_{\psi H} g_{\mathcal{N N} H}}{m_{H}^{2}}\right)^{2}
$$

for momentum transfers small relative to $m_{h, H}$. Given that the Higgs-nucleon coupling $g_{\mathcal{N N} \mathcal{H}}$, for $\mathcal{N}=p$ or $n$ and $\mathcal{H}=h$ or $H$, depends on $k_{u, d}^{\mathcal{H}}$ according to eq. (3.10), it may be possible to suppress $g_{\mathcal{N N \mathcal { H }}}$ sufficiently with a suitable choice of $k_{d}^{\mathcal{H}} / k_{u}^{\mathcal{H}}$ to make $\sigma_{\text {el }}^{\mathcal{N}}$ evade its experimental limit [15], at least for some of the $m_{\psi}$ values. In addition, the $\lambda_{h, H}$ terms in eq. (3.18) may (partially) cancel each other to lower $\sigma_{\text {el }}^{\mathcal{N}}$ as well. These are appealing features of the two-Higgs-doublet scenario that the one-doublet case does not possess. In evaluating model predictions for DM-nucleon reactions later on, we work exclusively with the DM-proton cross-section, $\sigma_{\mathrm{el}}^{p}$, and then convert it to $\sigma_{\mathrm{el}}^{N}$ with the aid of eq. (3.11) for comparison with measurements.

As there are countless different possibilities in which $h$ and $H$ may act as portals between the DM and other particles, for definiteness and simplicity hereafter we concentrate on two scenarios in which the 125-GeV Higgs boson $h$ is lighter than the other Higgs bosons, $m_{h}<m_{H, A, H^{ \pm}}$. Moreover, we assume particularly that either $H$ or $h$ has a vanishing coupling to the DM, $\lambda_{\psi H}=0$ or $\lambda_{\psi h}=0$, respectively. Accordingly, either $h$ or $H$ alone serves as the portal, and hence now we have $f_{n} / f_{p}=g_{n n \mathcal{H}} / g_{p p \mathcal{H}}$, after ignoring the $n$ - $p$ mass difference.

In the $h$-portal scenario $\left(\lambda_{\psi H}=0\right)$, the cross section of DM annihilation is

$$
\sigma_{\mathrm{ann}}=\sigma\left(\bar{\psi} \psi \rightarrow h^{*} \rightarrow X_{\mathrm{SM}}\right)+\sum_{\mathbf{s}_{1} \mathbf{s}_{2}} \sigma\left(\bar{\psi} \psi \rightarrow \mathbf{s}_{1} \mathbf{s}_{2}\right),
$$

where $\sigma\left(\bar{\psi} \psi \rightarrow h^{*} \rightarrow X_{\mathrm{SM}}\right)$ is equal to that in eq. (2.3), except the couplings of $h$ to fermions and gauge bosons are multiplied by the appropriate $k_{u, d, V}^{h}$ factors mentioned earlier, the sum is over $\mathbf{s}_{1} \mathbf{s}_{2}=h h, h H, H H, A A, H^{+} H^{-}$with only kinematically permitted channels contributing, and the formulas for $\sigma\left(\bar{\psi} \psi \rightarrow \mathbf{s}_{1} \mathbf{s}_{2}\right)$ are listed in to appendix A. After extracting $\lambda_{\psi h}$ from the relic density data and evaluating $g_{p p h}$ with the $\alpha$ and $\beta$ choices consistent with eq. (3.9), we can predict the cross section of the $h$-mediated transition $\psi p \rightarrow \psi p$,

$$
\sigma_{\mathrm{el}}^{p}=\frac{\lambda_{\psi h}^{2} g_{p p h}^{2} m_{\psi}^{2} m_{p}^{2}}{\pi\left(m_{\psi}+m_{p}\right)^{2} m_{h}^{4}} .
$$

As in section 2.1, for $2 m_{\psi}<m_{h}$ the invisible channel $h \rightarrow \bar{\psi} \psi$ is open, its rate already written down in eq. (2.4). Then $\mathcal{B}(h \rightarrow \bar{\psi} \psi)$ must be consistent with the LHC information on the Higgs invisible decay, and so for this $m_{\psi}$ range we again impose the bound in eq. (2.5).

In the $H$-portal case $\left(\lambda_{\psi h}=0\right)$, the DM-annihilation cross-section is

$$
\sigma_{\mathrm{ann}}=\sigma\left(\bar{\psi} \psi \rightarrow H^{*} \rightarrow X_{\mathrm{SM}}\right)+\sum_{\mathbf{s}_{1} \mathbf{s}_{2}} \sigma\left(\bar{\psi} \psi \rightarrow \mathbf{s}_{1} \mathbf{s}_{2}\right)
$$




\begin{tabular}{|c|cccccc|ccccccccc|}
\hline Set & $\alpha$ & $\beta$ & $\frac{m_{H}}{\mathrm{GeV}}$ & $\frac{m_{A}}{\mathrm{GeV}}$ & $\frac{m_{H^{ \pm}}}{\mathrm{GeV}}$ & $\frac{m_{12}^{2}}{\mathrm{GeV}^{2}}$ & $k_{V}^{h}$ & $k_{u}^{h}$ & $\frac{k_{d}^{h}}{k_{u}^{h}}$ & $k_{V}^{H}$ & $k_{u}^{H}$ & $k_{d}^{H}$ & $\frac{g_{p p h}}{10^{-5}}$ & $\frac{f_{n}}{f_{p}}$ \\
\hline 1 & 0.141 & 1.422 & 550 & 520 & 540 & 44000 & 0.958 & 1.001 & -0.947 & 0.286 & 0.142 & 6.68 & 3.29 & -0.197 \\
2 & 0.206 & 1.357 & 515 & 560 & 570 & 55000 & 0.913 & 1.002 & -0.962 & 0.408 & 0.209 & 4.61 & 2.42 & -0.646 \\
\hline
\end{tabular}

Table 1. Sample values of input parameters $\alpha, \beta, m_{H, A, H^{ \pm}}$, and $m_{12}^{2}$ in the $h$-portal scenarios $\left(\lambda_{\psi H}=\lambda_{\Psi H}=\lambda_{H}=0\right)$ and the resulting values of several quantities, including $f_{n} / f_{p}=g_{n n h} / g_{p p h}$.

\begin{tabular}{|c|cccccc|ccccccccc|}
\hline Set & $\alpha$ & $\beta$ & $\frac{m_{H}}{\mathrm{GeV}}$ & $\frac{m_{A}}{\mathrm{GeV}}$ & $\frac{m_{H^{ \pm}}}{\mathrm{GeV}}$ & $\frac{m_{12}^{2}}{\mathrm{GeV}^{2}}$ & $k_{V}^{h}$ & $k_{u}^{h}$ & $k_{d}^{h}$ & $k_{V}^{H}$ & $k_{u}^{H}$ & $\frac{k_{d}^{H}}{k_{u}^{H}}$ & $\frac{g_{p p H}}{10^{-5}}$ & $\frac{f_{n}}{f_{p}}$ \\
\hline 3 & -0.749 & 0.723 & 610 & 750 & 760 & 91000 & 0.995 & 1.107 & 0.908 & 0.099 & -1.029 & -0.949 & -3.26 & -0.245 \\
4 & -0.676 & 0.658 & 590 & 610 & 640 & 60000 & 0.972 & 1.276 & 0.791 & 0.235 & -1.023 & -0.964 & -2.40 & -0.693 \\
\hline
\end{tabular}

Table 2. The same as table 1 , but for the $H$-portal scenarios $\left(\lambda_{\psi h}=\lambda_{\Psi h}=\lambda_{h}=0\right)$.

where

$$
\sigma\left(\bar{\psi} \psi \rightarrow H^{*} \rightarrow X_{\mathrm{SM}}\right)=\frac{\beta_{\psi} \lambda_{\psi H}^{2} \sqrt{s} \sum_{i} \Gamma\left(\tilde{H} \rightarrow X_{i, \mathrm{SM}}\right)}{2\left[\left(m_{H}^{2}-s\right)^{2}+\Gamma_{H}^{2} m_{H}^{2}\right]},
$$

with $\tilde{H}$ being a virtual $H$ with mass $m_{\tilde{H}}=\sqrt{s}$. Given that $H$ is not yet discovered, no empirical restraint on $H \rightarrow \bar{\psi} \psi$ exists. For the $\psi$-proton scattering via $H$ exchange, the cross section is

$$
\sigma_{\mathrm{el}}^{p}=\frac{\lambda_{\psi H}^{2} g_{p p H}^{2} m_{\psi}^{2} m_{p}^{2}}{\pi\left(m_{\psi}+m_{p}\right)^{2} m_{H}^{4}} .
$$

In applying eq. (3.11), we set $f_{n} / f_{p}=g_{n n H} / g_{p p H}$.

To illustrate the viable parameter space in these $\lambda_{\psi H(\psi h)}=0$ scenarios, we put together in table 1 (2) sample sets of input parameters (the second to seventh columns) which are compatible with eq. (3.13) and the other requirements described in the two paragraphs following it. The eighth to fifteenth columns of the tables contain the resulting values of several quantities.

With these input numbers, we show in figures 4(a) and 4(b) the $\lambda_{\psi h}$ and $\lambda_{\psi H}$ regions evaluated from the observed relic density. One observes that the $\left|\lambda_{\psi H}\right|$ values extracted from the relic density data tend to be bigger than their $\lambda_{\psi h}$ counterparts. This is because the $H$-portal annihilation rate is relatively more suppressed due to $m_{H}>m_{h}$. In figure 4(a), we also display the upper bound on $\left|\lambda_{\psi h}\right|$ inferred from eq. (2.5) for the $h$ invisible decay (black dotted curve). Like in the minimal model of section 2.1, the limited extent of the reliability of the EFT approximation for the $\psi$-Higgs operators in $\mathcal{L}_{\psi}^{\prime}$ implies that in each $\mathcal{H}$-portal instance we also need to ensure $\left|\lambda_{\psi \mathcal{H}}\right|<v / m_{\psi}$ beyond which the EFT framework may be expected to break down. This condition is represented by the lower sides of the magenta bands in figures 4(a) and 4(b). We exhibit the corresponding predictions for $\sigma_{\mathrm{el}}^{p}$ in figure 4(c), where the dotted parts of the green and blue curves are excluded by the constraints in figures $4(\mathrm{a})$ and $4(\mathrm{~b})$, respectively.

To test the model with direct search results, which are typically reported in terms of the DM-nucleon cross-section $\sigma_{\mathrm{el}}^{N}$, we have converted the calculated $\sigma_{\mathrm{el}}^{p}$ in figure 4 to the 

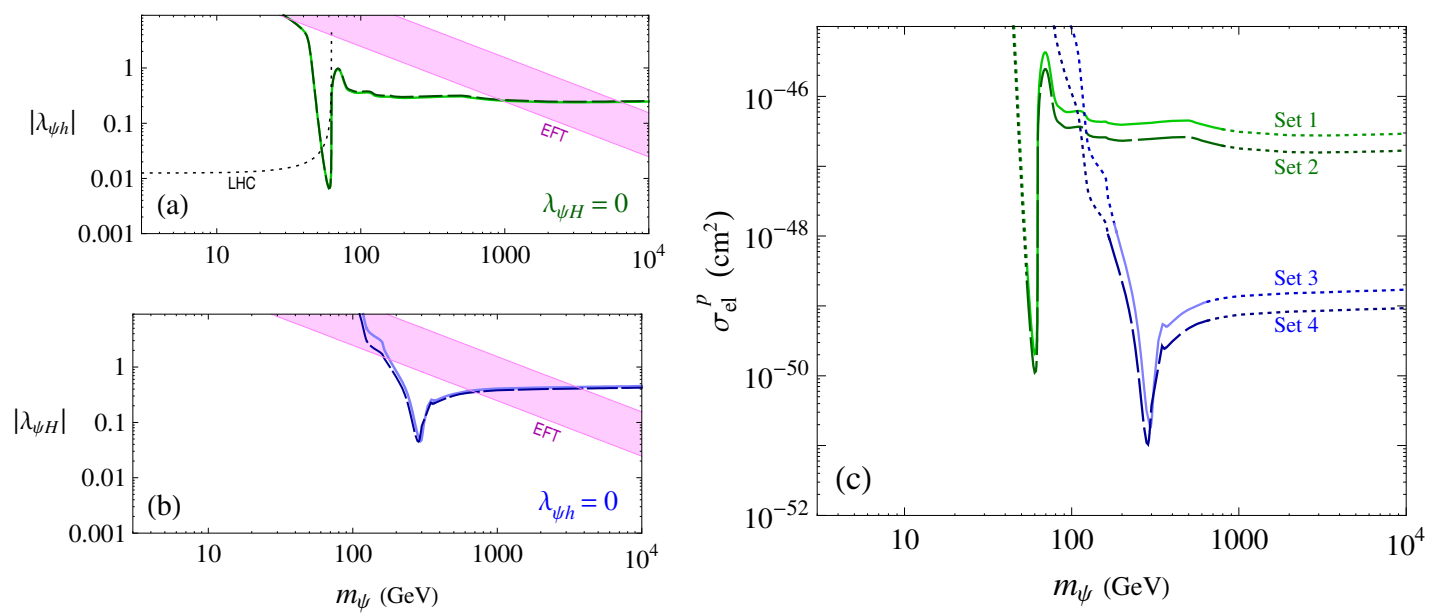

Figure 4. (a) The magnitude of DM- $h$ coupling $\lambda_{\psi h}$ consistent with the relic density data versus $m_{\psi}$ in the THDM II $+\psi$ with $\lambda_{\psi H}=0$ and input numbers from Sets 1 (green solid curve) and 2 (green dashed curve) in table 1. Also plotted are upper limits inferred from LHC data on the Higgs invisible decay (black dotted curve) and from the validity of the EFT approximation (lower sides of magenta bands). (b) The same as (a), except with $\lambda_{\psi h}=0$ and input numbers from Sets 3 (blue solid curve) and 4 (blue dashed curve) in table 2, but without the $h$ invisible decay restraint. (c) The corresponding $\psi$-proton cross-sections $\sigma_{\mathrm{el}}^{p}$. The dotted portions are disallowed by the constraints in (a) and (b).

(green and blue) $\sigma_{\text {el }}^{N}$ curves in figure $5\left(\right.$ a) by means of eq. (3.11) with the $f_{n} / f_{p}$ values from the tables, assuming that the target material in the detector is xenon. Since the DarkSide G2 experiment will employ an argon target [66], in figure 5(b) we plot the corresponding predictions for $\sigma_{\text {el }}^{N}$ assuming an argon target instead. These graphs reveal some visible differences, especially the xenon curves for Sets 2 and 4 which are significantly lower than their argon counterparts. The differences are not unexpected because the $f_{n} / f_{p}$ numbers in these instances are not far from the xenophobic extreme, $f_{n} / f_{p} \simeq-0.7$.

Also depicted in figure 5 are the same data and projections as in figure 1(b). It is obvious that, in stark contrast to the $\mathrm{SM}+\psi$, the THDM II $+\psi$ accommodates a good amount of parameter space which can evade the current direct search restrictions very well. Particularly in these examples, over wide stretches of $m_{\psi}$ the model prediction can also escape future direct detection and even hide below the neutrino floor. In the $h$-portal cases, the LHC Higgs invisible decay data and the EFT validity limit rule out $m_{\psi}<54 \mathrm{GeV}$ and $m_{\psi}>0.9 \mathrm{TeV}$, respectively. In the $H$-portal ones, the LHC Higgs invisible decay restraint does not apply, but the EFT validity limit disallows $m_{\psi}$ values below $165 \mathrm{GeV}$ and above $0.7 \mathrm{TeV}$.

\subsection{THDM II $+\Psi$}

In this model, the Lagrangian for the spin-3/2 DM is

$$
\mathcal{L}_{\Psi}^{\prime}=-\overline{\Psi_{\nu}}\left(i \not \partial-\mu_{\Psi}\right) \Psi^{\nu}+\overline{\Psi_{\nu}} \Psi^{\nu}\left(\frac{H_{1}^{\dagger} H_{1}}{\Lambda_{1 \Psi}}+\frac{H_{2}^{\dagger} H_{2}}{\Lambda_{2 \Psi}}\right),
$$



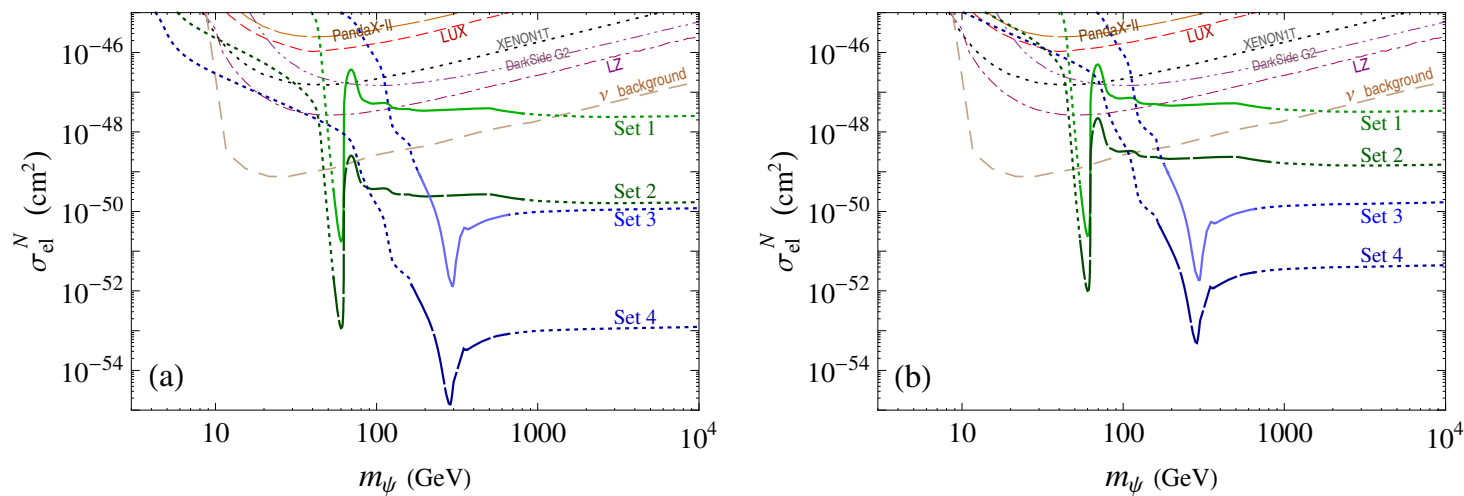

Figure 5. The predicted DM-nucleon cross-sections in the THDM II+ $\psi$ with input numbers from Sets 1 and 2 (green curves) in table 1 and Sets 3 and 4 (blue curves) in tables 2 for (a) xenon and (b) argon targets, compared to the same data and projections as in figure 1(b). The dotted portions of the green and blue curves are excluded as in figure 4.

where $\mu_{\Psi}$ and $\Lambda_{1 \Psi, 2 \Psi}$ are real constants of dimension mass and the latter two contain the parameters of the underlying heavy new physics. After electroweak symmetry breaking, we can then express the relevant terms in $\mathcal{L}_{\Psi}^{\prime}$ involving the physical bosons as

$$
\begin{aligned}
\mathcal{L}_{\Psi}^{\prime} \supset & m_{\Psi} \overline{\Psi_{\nu}} \Psi^{\nu}+\overline{\Psi_{\nu}} \Psi^{\nu}\left(\lambda_{\Psi h} h+\lambda_{\Psi H} H\right) \\
& +\frac{\overline{\Psi_{\nu}} \Psi^{\nu}}{2 v}\left(\lambda_{\Psi h h} h^{2}+2 \lambda_{\Psi h H} h H+\lambda_{\Psi H H} H^{2}+\lambda_{\Psi A A} A^{2}+2 \lambda_{\Psi H^{+} H^{-}} H^{+} H^{-}\right),
\end{aligned}
$$

where $m_{\Psi}$ and the $\lambda_{\mathrm{s}}$ are the same in form as their counterparts in eqs. (3.16) and (3.17), but with $\psi$ in the subscripts replaced by $\Psi$.

It follows that the DM-annihilation cross-section in the $h$-portal scenario is

$$
\sigma_{\mathrm{ann}}=\sigma\left(\bar{\Psi} \Psi \rightarrow h^{*} \rightarrow X_{\mathrm{SM}}\right)+\sum_{\mathrm{s}_{1} \mathrm{~s}_{2}} \sigma\left(\bar{\Psi} \Psi \rightarrow \mathrm{s}_{1} \mathrm{~s}_{2}\right)
$$

where $\sigma\left(\bar{\Psi} \Psi \rightarrow h^{*} \rightarrow X_{\mathrm{SM}}\right)$ is equal to that in eq. (2.9), except the $h$ couplings of to $\mathrm{SM}$ particles are scaled by the suitable $k_{u, d, V}^{h}$ factors, the sum is again over $\mathbf{s}_{1} \mathbf{s}_{2}=$ $h h, h H, H H, A A, H^{+} H^{-}$, and the formulas for $\sigma\left(\bar{\Psi} \Psi \rightarrow \mathbf{s}_{1} \mathbf{s}_{2}\right)$ are collected in appendix A. As in section 2.2, for $2 m_{\Psi}<m_{h}$ the invisible channel $h \rightarrow \bar{\Psi} \Psi$ is open, its rate given by eq. (2.10), and so it must fulfill the condition in eq. (2.11). The $\Psi$-proton cross-section is the same as that in eq. (3.20) but with $\psi$ in the subscripts replaced by $\Psi$.

In the $H$-portal scenario

$$
\sigma_{\mathrm{ann}}=\sigma\left(\bar{\Psi} \Psi \rightarrow H^{*} \rightarrow X_{\mathrm{SM}}\right)+\sum_{\mathbf{s}_{1} \mathbf{s}_{2}} \sigma\left(\bar{\Psi} \Psi \rightarrow \mathbf{s}_{1} \mathbf{s}_{2}\right)
$$

where

$$
\sigma\left(\bar{\Psi} \Psi \rightarrow H^{*} \rightarrow X_{\mathrm{SM}}\right)=\frac{\left(5 \beta_{\Psi}-6 \beta_{\Psi}^{3}+9 \beta_{\Psi}^{5}\right) \lambda_{\Psi H}^{2} s^{5 / 2} \sum_{i} \Gamma\left(\tilde{H} \rightarrow X_{i, \mathrm{SM}}\right)}{576 m_{\Psi}^{4}\left[\left(m_{H}^{2}-s\right)^{2}+\Gamma_{H}^{2} m_{H}^{2}\right]} .
$$

The cross section of $H$-mediated $\Psi$-proton scattering is equal to that in eq. (3.23), but with $\psi$ in the subscripts replaced by $\Psi$. 

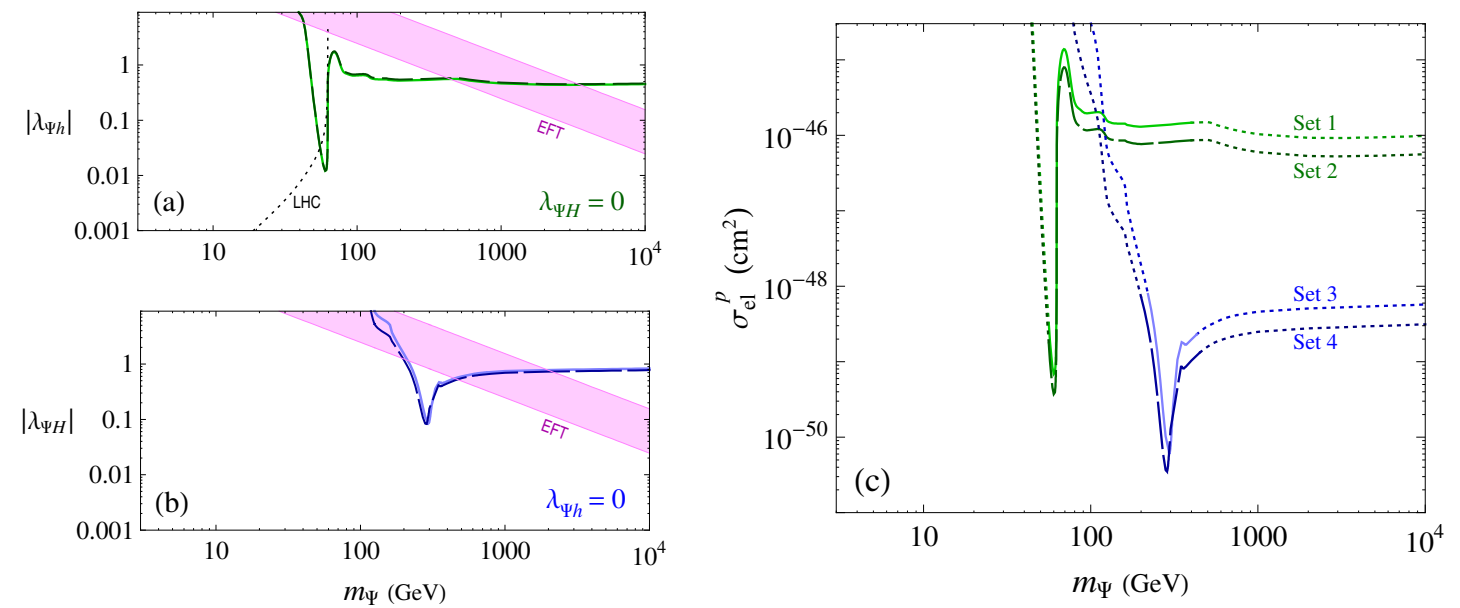

Figure 6. The same as figure 4 , except the DM is the spin- $3 / 2$ singlet $\Psi$ in the THDM II $+\Psi$.
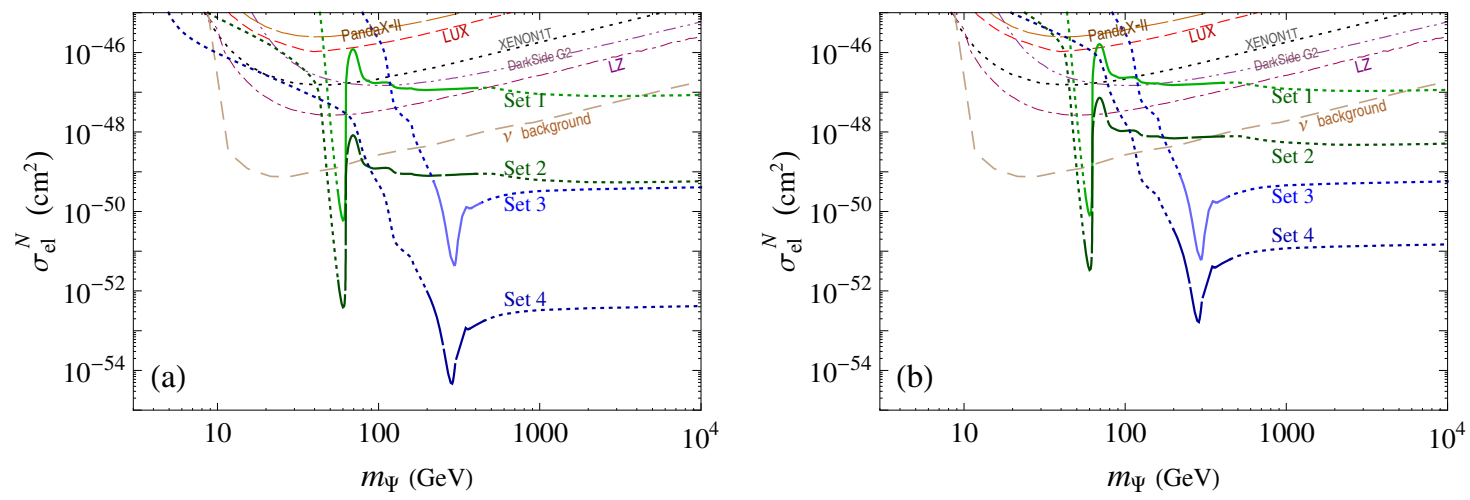

Figure 7. The same as figure 5 , except the DM is the spin- $3 / 2$ singlet $\Psi$ in the THDM II $+\Psi$.

Similarly to the THDM II $+\psi$, we use the numbers from tables 1 and 2 for our examples. We present the results in figures 6 and 7 . These instances indicate that in the THDM II $+\Psi$ the situation is roughly similar to that in the THDM II $+\psi$, but the parameter space in the former is less able than in the latter to escape the different restrictions. Specifically for these examples, we estimate the viable zones in the $h$ - and $H$-portal cases, respectively, to be $56 \mathrm{GeV} \lesssim m_{\Psi} \lesssim 420 \mathrm{GeV}$ and $200 \mathrm{GeV} \lesssim m_{\Psi} \lesssim 450 \mathrm{GeV}$, which translate into predictions for $\sigma_{\mathrm{el}}^{N}$ which are currently below its experimental limits.

\subsection{THDM II+V}

In this scenario, the Lagrangian for the vector DM is [32]

$$
\begin{aligned}
\mathcal{L}_{V}^{\prime}= & -\frac{1}{4} V_{\kappa \nu} V^{\kappa \nu}+\frac{\mu_{V}^{2}}{2} V_{\kappa} V^{\kappa}+\frac{\lambda_{V}}{4}\left(V_{\kappa} V^{\kappa}\right)^{2}+\left(\lambda_{1 \mathrm{v}} H_{1}^{\dagger} H_{1}+\lambda_{2 \mathrm{v}} H_{2}^{\dagger} H_{2}\right) V_{\kappa} V^{\kappa} \\
\supset & \frac{m_{V}^{2}}{2} V_{\kappa} V^{\kappa}+\left(\lambda_{h} h+\lambda_{H} H\right) V_{\kappa} V^{\kappa} v \\
& +\frac{1}{2}\left(\lambda_{h h} h^{2}+2 \lambda_{h H} h H+\lambda_{H H} H^{2}+\lambda_{A A} A^{2}+2 \lambda_{H^{+} H^{-}} H^{+} H^{-}\right) V_{\kappa} V^{\kappa},
\end{aligned}
$$


where, analogously to the fermionic models,

$$
\begin{aligned}
m_{V}^{2} & =\mu_{V}^{2}+\left(\lambda_{1 \mathrm{v}} c_{\beta}^{2}+\lambda_{2 \mathrm{v}} s_{\beta}^{2}\right) v^{2}, & \lambda_{h} & =\lambda_{2 \mathrm{~V}} c_{\alpha} s_{\beta}-\lambda_{1 \mathrm{v}} s_{\alpha} c_{\beta}, \\
\lambda_{H} & =\lambda_{1 \mathrm{v}} c_{\alpha} c_{\beta}+\lambda_{2 \mathrm{v}} s_{\alpha} s_{\beta}, & \lambda_{H H} & =\left(\frac{c_{\alpha}^{3}}{c_{\beta}}+\frac{s_{\alpha}^{3}}{s_{\beta}}\right) \lambda_{H}-\frac{s_{2 \alpha} s_{\beta-\alpha}}{s_{2 \beta}} \lambda_{h}, \\
\lambda_{h h} & =\left(\frac{c_{\alpha}^{3}}{s_{\beta}}-\frac{s_{\alpha}^{3}}{c_{\beta}}\right) \lambda_{h}+\frac{s_{2 \alpha} c_{\beta-\alpha}}{s_{2 \beta}} \lambda_{H}, & \lambda_{H} & s_{\alpha} \\
\lambda_{h H} & =\frac{s_{2 \alpha}}{s_{2 \beta}}\left(\lambda_{h} c_{\beta-\alpha}-\lambda_{H} s_{\beta-\alpha}\right), & \lambda_{A A, H^{+} H^{-}} & =\frac{c_{\alpha} c_{\beta}^{3}-s_{\alpha} s_{\beta}^{3}}{c_{\beta} s_{\beta}} \lambda_{h}+\frac{c_{\alpha} s_{\beta}^{3}+s_{\alpha} c_{\beta}^{3}}{c_{\beta} s_{\beta}} \lambda_{H} .
\end{aligned}
$$

We again look at separate possibilities with either $\lambda_{H}=0$ or $\lambda_{h}=0$.

Thus, the cross section of the $h$-portal DM-annihilation is

$$
\sigma_{\mathrm{ann}}=\sigma\left(\mathrm{VV} \rightarrow h^{*} \rightarrow X_{\mathrm{SM}}\right)+\sum_{\mathrm{s}_{1} \mathbf{s}_{2}} \sigma\left(\mathrm{VV} \rightarrow \mathbf{s}_{1} \mathbf{s}_{2}\right),
$$

where $\sigma\left(V V \rightarrow h^{*} \rightarrow X_{\mathrm{SM}}\right)$ equals that in eq. (2.15), but with the $h$ couplings to SM members multiplied by the proper $k_{u, d, V}^{h}$ factors. The expressions for $\sigma\left(V V \rightarrow \mathbf{s}_{1} \mathbf{s}_{2}\right)$ can be found in appendix A. The invisible channel $h \rightarrow V V$ has the rate already given in eq. (2.16) and hence is also constrained by eq. (2.17). For the $h$-mediated DM-nucleon collision $V N \rightarrow V N$, the cross section is

$$
\sigma_{\mathrm{el}}^{p}=\frac{\lambda_{h}^{2} g_{p p h}^{2} m_{p}^{2} v^{2}}{\pi\left(m_{V}+m_{p}\right)^{2} m_{h}^{4}} .
$$

In the $H$-portal scenario, the cross section of DM annihilation is

$$
\sigma_{\mathrm{ann}}=\sigma\left(V V \rightarrow H^{*} \rightarrow X_{\mathrm{SM}}\right)+\sum_{\mathrm{s}_{1} \mathbf{s}_{2}} \sigma\left(V V \rightarrow \mathrm{s}_{1} \mathbf{s}_{2}\right)
$$

where

$$
\sigma\left(V V \rightarrow H^{*} \rightarrow X_{\mathrm{SM}}\right)=\frac{\lambda_{H}^{2}\left(\beta_{V}^{2} s^{2}+12 m_{V}^{4}\right) v^{2} \sum_{i} \Gamma\left(\tilde{H} \rightarrow X_{i, \mathrm{SM}}\right)}{9 \beta_{V} m_{V}^{4} \sqrt{s}\left[\left(m_{H}^{2}-s\right)^{2}+\Gamma_{H}^{2} m_{H}^{2}\right]} .
$$

The cross section of the $H$-mediated $V N \rightarrow V N$ scattering has the formula in eq. (3.32), but with $h$ in the subscripts replaced by $H$.

Employing these formulas with the input numbers from tables 1 and 2 for our examples, we arrive at the green and blue curves in figures 8 and 9 . In figure $8(\mathrm{a})$, we also draw the upper bound on $\left|\lambda_{h}\right|$ from the Higgs data (black dotted curve). As in the SM+V, theoretical considerations concerning unitarity and perturbativity lead to the constraints $\left|\lambda_{\mathcal{H}}\right|<\sqrt{2 \pi} m_{V} / v$ and $\left|\lambda_{\mathcal{H}}\right|<1$, respectively, which are represented by the magenta dashed lines in figure 8 . These instances illustrate that the situation in this model is roughly similar to that in the THDM II plus real scalar DM investigated in ref. [9]. Specifically, in our $h$-portal examples the LHC constraint on $h \rightarrow V V$ rules out $m_{V}<54 \mathrm{GeV}$ and the effective theory is likely to be no longer perturbative for $m_{V}>5 \mathrm{TeV}$, whereas in the 

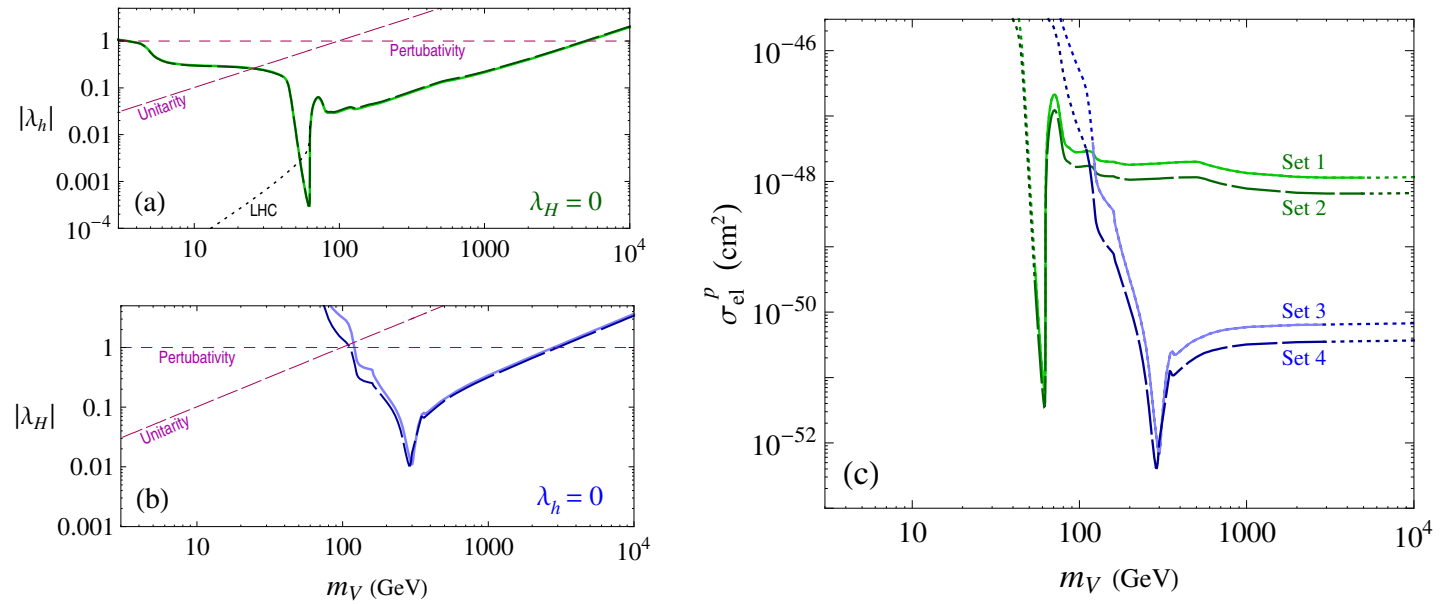

Figure 8. The same as figure 4, except the DM is the spin-1 singlet $V$ in the THDM II+V with (a) $\lambda_{H}=0$ and (b) $\lambda_{h}=0$.
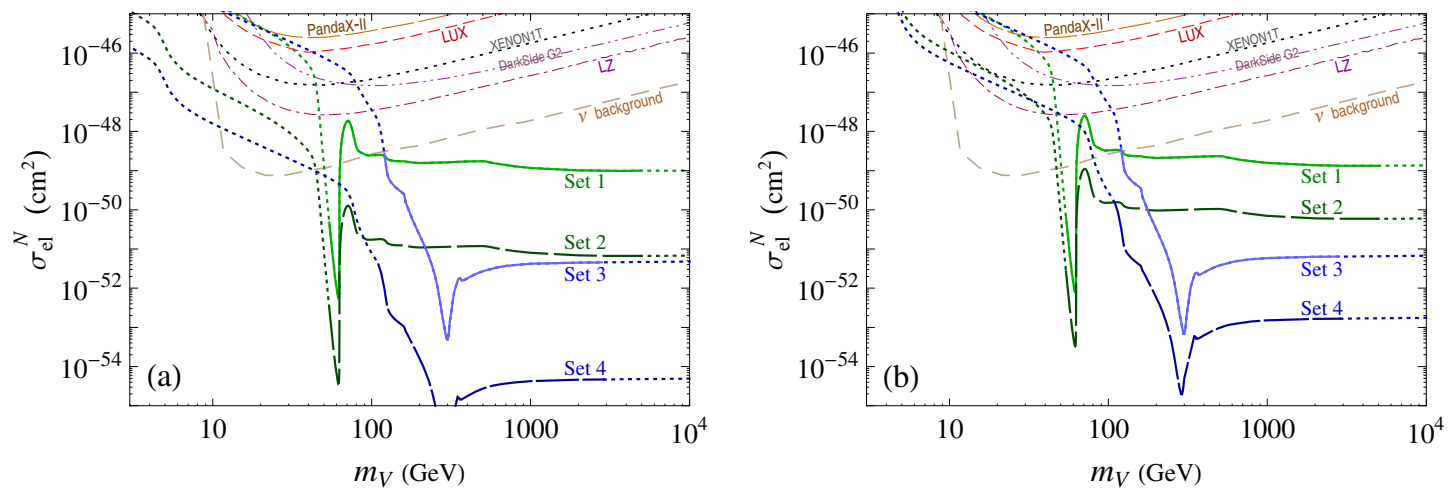

Figure 9. The predicted DM-nucleon cross-sections (green curves) in the THDM II+V with input numbers from Sets 1 and 2 (green curves) in table 1 and Sets 3 and 4 (blue curves) in tables 2 for (a) xenon and (b) argon targets, compared to the same data and projections as in figure 1(b). The dotted portions of the green and blue curves are disallowed as in figure 8.

$H$-portal cases the unitarity and perturbativity conditions disfavor $m_{V}<110 \mathrm{GeV}$ and $m_{V}>3 \mathrm{TeV}$. Thus, more generally, in great contrast to the THDM $\mathrm{II}+(\psi, \Psi)$ as well as the $\mathrm{SM}+\mathrm{V}$, the THDM II $+\mathrm{V}$ has plentiful parameter space that is far away from the current direct search limits and can even escape future ones.

\section{Conclusions}

The most recent limits from LUX and PandaX-II on DM-nucleon interactions and the available LHC data on the 125-GeV Higgs boson's couplings have led to strong restrictions on the simplest Higgs-portal dark matter models. Taking these constraints into account, we have revisited the minimal models with fermionic DM having spin $1 / 2$ or $3 / 2$ and a purely scalar effective coupling to the Higgs doublet. Realizing also that the EFT description for this coupling has limitations, we have found that these minimal fermionic models 
are ruled out except in narrow regions of the DM mass in the neighborhood of the Higgs resonance point at $m_{h} / 2$. On the other hand, the simplest Higgs-portal vector-DM model is viable not only around $m_{V}=m_{h} / 2$, but also above $m_{V} \sim 1.4 \mathrm{TeV}$, although it may lose perturbativity if $m_{V}>3.9 \mathrm{TeV}$. Slightly expanding each of these models with the addition of another Higgs doublet and assuming the extended Yukawa sector to be that of the type-II two-Higgs-doublet model, we can significantly relax the restraints from direct search and LHC data, even in the fermionic DM scenarios, and recover sizable parts of the regions excluded in the minimal models. This is due to suppression of the effective interactions of the portal $C P$-even Higgs bosons with nucleons at some values of the ratios of the Higgs couplings to the up and down quarks, rendering the interactions considerably isospin-violating. Sizable portions of the revived parameter space can yield a DM-nucleon scattering cross-section that is much smaller than its current experimental bound or even falls under the neutrino-background floor. Nevertheless, there are also areas in the parameter space of these two-Higgs-doublet-portal DM models that are still within the discovery reach of future quests such as XENON1T and LZ.

\section{Acknowledgments}

This work was supported in part by MOE Academic Excellence Program (Grant No. 105R891505) and NCTS of ROC. X.-G. He was also supported in part by MOST of ROC (Grant No. MOST104-2112-M-002-015-MY3) and in part by NSFC (Grant Nos. 11175115 and 11575111), Key Laboratory for Particle Physics, Astrophysics and Cosmology, Ministry of Education, and Shanghai Key Laboratory for Particle Physics and Cosmology (SKLPPC) (Grant No. 11DZ2260700) of PRC.

\section{A Extra formulas for DM reactions}

To extract the DM-Higgs coupling which enters the DM-annihilation cross-section $\sigma_{\text {ann }}$, we employ its thermal average [86]

$$
\left\langle\sigma v_{\mathrm{rel}}\right\rangle=\frac{x}{8 m_{\mathrm{DM}}^{5} K_{2}^{2}(x)} \int_{4 m_{\mathrm{DM}}^{2}}^{\infty} d s \sqrt{s}\left(s-4 m_{\mathrm{DM}}^{2}\right) K_{1}\left(\sqrt{s} x / m_{\mathrm{DM}}\right) \sigma_{\mathrm{ann}}
$$

where $v_{\text {rel }}$ is the relative speed of the DM pair, $K_{r}$ is the modified Bessel function of the second kind of order $r$ and $x$ can be set to its freeze-out value $x=x_{f}$, which is related to $\left\langle\sigma v_{\text {rel }}\right\rangle$ by $[87]$

$$
x_{f}=\ln \frac{0.038\left(2 J_{\mathrm{DM}}+1\right) m_{\mathrm{DM}} m_{\mathrm{Pl}}\left\langle\sigma v_{\mathrm{rel}}\right\rangle}{\sqrt{g_{*} x_{f}}},
$$

with $J_{\mathrm{DM}}$ being the DM particle's spin, $m_{\mathrm{Pl}}=1.22 \times 10^{19} \mathrm{GeV}$ the Planck mass, and $g_{*}$ the number of effectively relativistic degrees of freedom below the freeze-out temperature $T_{f}=m_{\mathrm{DM}} / x_{f}$. In addition, we adopt the numerical values of $\left\langle\sigma v_{\text {rel }}\right\rangle$ versus DM mass 

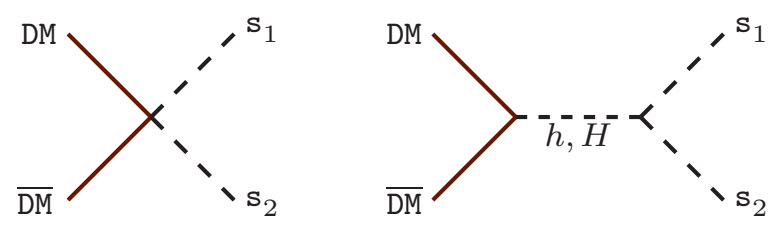

Figure 10. Feynman diagrams contributing at leading order to DM annihilation into $\mathbf{s}_{1} \mathbf{s}_{2}$.

determined in ref. [88], ${ }^{8}$ as well as the latest relic density data $\Omega \hat{h}^{2}=0.1197 \pm 0.0022[89]$, with $\hat{h}$ being the Hubble parameter.

In the THDMII $+\psi$, the DM annihilation mode $\bar{\psi} \psi \rightarrow \mathbf{s}_{1} \mathbf{s}_{2}$ can take place due to the diagrams displayed in figure 10 , where $\mathbf{s}_{1} \mathbf{s}_{2}=h h, h H, H H, A A, H^{+} H^{-}$. We ignore the contributions of $t$ - and $u$-channel $\psi$-mediated diagrams because they are at a higher order in $\lambda_{\psi \mathcal{H}}$ and of the same order as the potential contributions of next-to-leading effective operators not included in eq. (3.14). The cross sections of these reactions are then calculated to be

$$
\sigma\left(\bar{\psi} \psi \rightarrow \mathbf{s}_{1} \mathbf{s}_{2}\right)=\frac{\beta_{\psi} \tilde{\beta}_{\mathbf{s}_{1} \mathbf{s}_{2}}}{32\left(1+\delta_{\mathbf{s}_{1} \mathbf{s}_{2}}\right) \pi v^{2}}\left(\mathcal{R}_{\psi, \mathbf{s}_{1} \mathbf{s}_{2}}^{2}+\mathcal{I}_{\psi, \mathbf{s}_{1} \mathbf{s}_{2}}^{2}\right)
$$

where

$$
\begin{aligned}
\beta_{\mathrm{X}} & =\sqrt{1-\frac{4 m_{\mathrm{X}}^{2}}{s}}, \quad \tilde{\beta}_{\mathrm{s}_{1} \mathbf{s}_{2}}=\frac{\sqrt{\left(s-m_{\mathbf{s}_{1}}^{2}-m_{\mathrm{s}_{2}}^{2}\right)^{2}-4 m_{\mathbf{s}_{1}}^{2} m_{\mathrm{s}_{2}}^{2}}}{s}, \\
\mathcal{R}_{\psi, \mathrm{XY}} & =\lambda_{\psi \mathrm{XY}}+\frac{\lambda_{\psi h} \lambda_{h \mathrm{XY}}\left(s-m_{h}^{2}\right) v^{2}}{\left(s-m_{h}^{2}\right)^{2}+\Gamma_{h}^{2} m_{h}^{2}}+\frac{\lambda_{\psi H} \lambda_{H \mathrm{XY}}\left(s-m_{H}^{2}\right) v^{2}}{\left(s-m_{H}^{2}\right)^{2}+\Gamma_{H}^{2} m_{H}^{2}}, \\
\mathcal{I}_{\psi, \mathrm{XY}} & =\frac{\lambda_{\psi h} \lambda_{h \mathrm{XY}} \Gamma_{h} m_{h} v^{2}}{\left(s-m_{h}^{2}\right)^{2}+\Gamma_{h}^{2} m_{h}^{2}}+\frac{\lambda_{\psi H} \lambda_{H \mathrm{XY}} \Gamma_{H} m_{H} v^{2}}{\left(s-m_{H}^{2}\right)^{2}+\Gamma_{H}^{2} m_{H}^{2}},
\end{aligned}
$$

with $\lambda_{\psi \mathrm{xY}}$ being connected to $\lambda_{\psi \mathcal{H}}$ for $\mathcal{H}=h, H$ by eq. (3.17) and the expressions for the Higgs cubic couplings $\lambda_{\mathcal{H} X Y}$ listed in ref. [9]. In our scenarios of interest, $\Gamma_{H}$ gets contributions not only from the partial rates of $H$ decays into fermions and gauge bosons, analogously to $\Gamma_{h}$, but also from

$$
\Gamma(H \rightarrow \bar{\psi} \psi)=\frac{\lambda_{\psi H}^{2} m_{H}}{8 \pi}\left(1-\frac{4 m_{\psi}^{2}}{m_{H}^{2}}\right)^{3 / 2}, \quad \Gamma(H \rightarrow h h)=\frac{\lambda_{h h H}^{2} v^{2}}{8 \pi m_{H}} \sqrt{1-\frac{4 m_{h}^{2}}{m_{H}^{2}}}
$$

once these channels are open. The expression for $\sigma(\bar{\psi} \psi \rightarrow h h)$ in eq. (A.3) is applicable to that in eq. (2.3) belonging to the $\mathrm{SM}+\psi$, in which case there is only one coupling for the $\psi$-Higgs interaction, $\lambda_{\psi h h}=\lambda_{\psi h}$, and $H$ is absent, $\lambda_{\psi H}=\lambda_{h h H}=0$.

In the spin-3/2 DM model, THDM II $+\Psi$, the counterpart of eq. (A.3) is

$$
\sigma\left(\bar{\Psi} \Psi \rightarrow \mathbf{s}_{1} \mathbf{s}_{2}\right)=\frac{\left(5 \beta_{\Psi}-6 \beta_{\Psi}^{3}+9 \beta_{\Psi}^{5}\right) \tilde{\beta}_{\mathbf{s}_{1} \mathbf{s}_{2}} s^{2}}{9216\left(1+\delta_{\mathbf{s}_{1} \mathbf{s}_{2}}\right) \pi m_{\Psi}^{4} v^{2}}\left(\mathcal{R}_{\Psi, \mathbf{s}_{1} \mathbf{s}_{2}}^{2}+\mathcal{I}_{\Psi, \mathbf{s}_{1} \mathbf{s}_{2}}^{2}\right)
$$

\footnotetext{
${ }^{8}$ Since our fermionic DM candidates $\left(\psi\right.$ and $\left.\Psi_{\nu}\right)$ are complex fields, the $\left\langle\sigma v_{\text {rel }}\right\rangle$ values used in (A.1) and (A.2) for the fermionic cases are twice those provided by [88], which applied to real or self-conjugate DM.
} 
where $\mathcal{R}_{\Psi, \mathrm{XY}}\left(\mathcal{I}_{\Psi, \mathrm{XY}}\right)$ is the same as $\mathcal{R}_{\psi, \mathrm{XY}}\left(\mathcal{I}_{\psi, \mathrm{XY}}\right)$ in eq. (A.4), except the label $\psi$ in $\lambda_{\psi \mathcal{H}}$ and $\lambda_{\psi \mathrm{XY}}$ is replaced by $\Psi$. The $\sigma(\bar{\Psi} \Psi \rightarrow h h)$ formula in eq. (A.6) becomes that in eq. (2.9) belonging to the $\mathrm{SM}+\Psi$ if we set $\lambda_{\Psi h h}=\lambda_{\Psi h}$ and $\lambda_{\Psi H}=\lambda_{h h H}=0$.

In the THDM II $+\mathrm{V}$, the annihilation of the vector DM into a pair of Higgs bosons, $V V \rightarrow \mathbf{s}_{1} \mathbf{s}_{2}$, is induced by contact and $s$-channel diagrams (figure 10), as the $t$ - and $u$ channel ones are of higher order in $\lambda_{h, H}$ and hence neglected. The cross section is then

$$
\sigma\left(V V \rightarrow \mathbf{s}_{1} \mathbf{s}_{2}\right)=\frac{\left(\beta_{V}^{2} s^{2}+12 m_{V}^{4}\right) \tilde{\beta}_{\mathbf{s}_{1} \mathbf{s}_{2}}}{144 \beta_{V}\left(1+\delta_{\mathbf{s}_{1} \mathbf{s}_{2}}\right) \pi m_{V}^{4} s}\left(\mathcal{R}_{\mathbf{s}_{1} \mathbf{s}_{2}}^{2}+\mathcal{I}_{\mathbf{s}_{1} \mathbf{s}_{2}}^{2}\right),
$$

where

$$
\begin{aligned}
& \mathcal{R}_{\mathrm{XY}}=\lambda_{\mathrm{XY}}+\frac{\lambda_{h} \lambda_{h \mathrm{XY}}\left(s-m_{h}^{2}\right) v^{2}}{\left(s-m_{h}^{2}\right)^{2}+\Gamma_{h}^{2} m_{h}^{2}}+\frac{\lambda_{H} \lambda_{H \mathrm{XY}}\left(s-m_{H}^{2}\right) v^{2}}{\left(s-m_{H}^{2}\right)^{2}+\Gamma_{H}^{2} m_{H}^{2}}, \\
& \mathcal{I}_{\mathrm{XY}}=\frac{\lambda_{h} \lambda_{h \mathrm{XY}} \Gamma_{h} m_{h} v^{2}}{\left(s-m_{h}^{2}\right)^{2}+\Gamma_{h}^{2} m_{h}^{2}}+\frac{\lambda_{H} \lambda_{H \mathrm{XY}} \Gamma_{H} m_{H} v^{2}}{\left(s-m_{H}^{2}\right)^{2}+\Gamma_{H}^{2} m_{H}^{2}} .
\end{aligned}
$$

The $\sigma(V V \rightarrow h h)$ expression in eq. (A.7) is applicable to the $\mathrm{SM}+\mathrm{V}$, in which case there is only one coupling for the $V$-Higgs interaction, $\lambda_{h h}=\lambda_{h}$, and $H$ is again absent, $\lambda_{H}=$ $\lambda_{h h H}=0$.

\section{B Conditions for perturbativity, vacuum stability, and tree-level unitarity}

The parameters of the scalar potential $\mathcal{V}_{H}$ in eq. (3.2) are subject to a number of theoretical constraints. The usual assumption that the scalar interactions are in the perturbative regime implies that $\left|\lambda_{1,2,3,4,5}\right| \leq 8 \pi[90]$. The requisite stability of $\mathcal{V}_{H}$ implies that it has to be bounded from below, entailing that

$$
\lambda_{1}>0, \quad \lambda_{2}>0, \quad \lambda_{3}+\min \left(0, \lambda_{4}-\left|\lambda_{5}\right|\right)>-\sqrt{\lambda_{1} \lambda_{2}} .
$$

Another important limitation is that the amplitudes for scalar-scalar scattering $s_{1} s_{2} \rightarrow$ $s_{3} s_{4}$ at high energies respect unitarity. This amounts to demanding that the combinations [82, 91, 92]

$$
\begin{aligned}
& \frac{3}{2}\left(\lambda_{1}+\lambda_{2}\right) \pm \sqrt{\frac{9}{4}\left(\lambda_{1}-\lambda_{2}\right)^{2}+\left(2 \lambda_{3}+\lambda_{4}\right)^{2}}, \quad \frac{1}{2}\left(\lambda_{1}+\lambda_{2}\right) \pm \sqrt{\frac{1}{4}\left(\lambda_{1}-\lambda_{2}\right)^{2}+\lambda_{4}^{2}}, \\
& \frac{1}{2}\left(\lambda_{1}+\lambda_{2}\right) \pm \sqrt{\frac{1}{4}\left(\lambda_{1}-\lambda_{2}\right)^{2}+\lambda_{5}^{2}}, \quad \lambda_{3}+2 \lambda_{4} \pm 3 \lambda_{5}, \quad \lambda_{3} \pm \lambda_{4}, \quad \lambda_{3} \pm \lambda_{5}
\end{aligned}
$$

each not exceed $8 \pi$ in magnitude. We implement the conditions in eqs. (B.1) and (B) employing the relations

$$
\begin{array}{ll}
\lambda_{1}=\frac{s_{\alpha}^{2} m_{h}^{2}+c_{\alpha}^{2} m_{H}^{2}}{c_{\beta}^{2} v^{2}}-\frac{s_{\beta} m_{12}^{2}}{c_{\beta}^{3} v^{2}}, & \lambda_{3}=\frac{s_{2 \alpha}}{s_{2 \beta}} \frac{m_{H}^{2}-m_{h}^{2}}{v^{2}}+\frac{2 m_{H^{ \pm}}^{2}}{v^{2}}-\frac{2 m_{12}^{2}}{s_{2 \beta} v^{2}}, \\
\lambda_{2}=\frac{c_{\alpha}^{2} m_{h}^{2}+s_{\alpha}^{2} m_{H}^{2}}{s_{\beta}^{2} v^{2}}-\frac{c_{\beta} m_{12}^{2}}{s_{\beta}^{3} v^{2}}, & \lambda_{4}=\frac{m_{A}^{2}-2 m_{H^{ \pm}}^{2}}{v^{2}}+\frac{2 m_{12}^{2}}{s_{2 \beta} v^{2}}, \quad \lambda_{5}=\frac{2 m_{12}^{2}}{s_{2 \beta} v^{2}}-\frac{m_{A}^{2}}{v^{2}}
\end{array}
$$


derived from $\mathcal{V}_{H}$. Upon specifying $\alpha$ and $\beta$, we can then take $m_{h, H, A, H^{ \pm}, 12}$ and $\lambda_{h, H}$ as the free parameters instead of $\lambda_{1,2,3,4,5}$. The expressions in eq. (B.3) are in agreement with those in the literature [93].

Since the vector-DM models in sections 2.3 and 3.3 are not renormalizable and violate unitarity, we need to impose unitarity restrictions on the DM couplings $\lambda_{h, H}$. Given that $\lambda_{h, H}$ and $\lambda_{V}$ are free parameters in our analysis, for simplicity we suppose that the DM self-coupling $\lambda_{V}$ is absent [77]. The amplitude for $V V \rightarrow V V$ at high energies, $\sqrt{s} \gg m_{V, h, H}$, is then

$$
\mathcal{M}_{V V \rightarrow V V}=\frac{4\left(\lambda_{h}^{2}+\lambda_{H}^{2}\right) v^{2}}{m_{V}^{2}}
$$

The unitarity condition implies that $\left|\mathcal{M}_{V V \rightarrow V V}\right|<8 \pi$, which translates into

$$
\lambda_{h}^{2}+\lambda_{H}^{2}<\frac{2 \pi m_{V}^{2}}{v^{2}} .
$$

In the $\mathrm{SM}+\mathrm{V}$, this becomes $\left|\lambda_{h}\right|<\sqrt{2 \pi} m_{V} / v$, which is used in section 2.3 .

There is a complementary theoretical restraint on $\lambda_{h}$ (or $\lambda_{H}$ ) having to do with the supposed perturbativity of the effective $V$ interactions. To get a rough estimate on the implied cap on $\lambda_{h}$, we may assume that $\lambda_{h} \mathrm{H}^{\dagger} \mathrm{H} V_{\kappa} V^{\kappa}$ in $\mathcal{L}_{V}$ arises from a tree-level diagram mediated by a heavy scalar $X$ coupled to $h$ and $V$ according to $\mathcal{L}_{X} \supset-g_{h} h^{2} X+g_{V} V_{\kappa} V^{\kappa} X$ in the UV-complete theory, as proposed in ref. [77]. We may further assume that $g_{h} \sim$ $\lambda_{h X} v_{X}$ and $g_{V} \sim m_{V}^{2} / v_{X}$, where $v_{X}$ is the VEV of $X$, in analogy to the scalar and weakboson couplings in the SM, ignoring potential modifications due to $h-X$ mixing. The EFT will then remain a reliable approach and perturbative if $2\left|\lambda_{h}\right| \sim 4\left|\lambda_{h X}\right| m_{V}^{2} / m_{X}^{2}<\left|\lambda_{h X}\right|<$ $4 \pi$, as the $s$-channel energy $\sqrt{s}$ satisfies $m_{X}^{2}>s>4 m_{V}^{2}$. Since it is likely that the EFT description starts to break down at a lower $\left|\lambda_{h X}\right|$, as was also suggested in section 2 for the fermionic cases, it is more reasonable to select $\left|\lambda_{h}\right|<1$ instead. Similarly, we impose $\left|\lambda_{H}\right|<1$ in the $H$-portal THDM II+V scenarios.

Open Access. This article is distributed under the terms of the Creative Commons Attribution License (CC-BY 4.0), which permits any use, distribution and reproduction in any medium, provided the original author(s) and source are credited.

\section{References}

[1] LUX collaboration, D.S. Akerib et al., Results from a search for dark matter in the complete LUX exposure, Phys. Rev. Lett. 118 (2017) 021303 [arXiv: 1608.07648] [INSPIRE].

[2] PandaX-II collaboration, A. Tan et al., Dark matter results from first 98.7 days of data from the PandaX-II experiment, Phys. Rev. Lett. 117 (2016) 121303 [arXiv:1607.07400] [INSPIRE].

[3] CRESST collaboration, G. Angloher et al., Results on light dark matter particles with a low-threshold CRESST-II detector, Eur. Phys. J. C 76 (2016) 25 [arXiv:1509.01515] [INSPIRE]. 
[4] SuperCDMS collaboration, R. Agnese et al., New results from the search for low-mass weakly interacting massive particles with the CDMS low ionization threshold experiment, Phys. Rev. Lett. 116 (2016) 071301 [arXiv: 1509.02448] [INSPIRE].

[5] P. Cushman et al., Working group report: WIMP dark matter direct detection, arXiv:1310.8327 [INSPIRE].

[6] ATLAS collaboration, Constraints on new phenomena via Higgs boson couplings and invisible decays with the ATLAS detector, JHEP 11 (2015) 206 [arXiv:1509.00672] [INSPIRE].

[7] CMS collaboration, Searches for invisible decays of the Higgs boson in pp collisions at $\sqrt{s}=7,8$ and $13 \mathrm{TeV}$, JHEP 02 (2017) 135 [arXiv:1610.09218] [INSPIRE].

[8] ATLAS, CMS collaboration, Measurements of the Higgs boson production and decay rates and constraints on its couplings from a combined ATLAS and CMS analysis of the LHC pp collision data at $\sqrt{s}=7$ and $8 \mathrm{TeV}$, JHEP 08 (2016) 045 [arXiv:1606.02266] [INSPIRE].

[9] X.-G. He and J. Tandean, New LUX and PandaX-II results illuminating the simplest Higgs-portal dark matter models, JHEP 12 (2016) 074 [arXiv: 1609.03551] [INSPIRE].

[10] M. Escudero, A. Berlin, D. Hooper and M.-X. Lin, Toward (finally!) ruling out $Z$ and Higgs mediated dark matter models, JCAP 12 (2016) 029 [arXiv: 1609.09079] [INSPIRE].

[11] H. Wu and S. Zheng, Scalar dark matter: real vs. complex, arXiv:1610.06292 [INSPIRE].

[12] GAMBIT collaboration, J.M. Cornell, Global fits of scalar singlet dark matter with GAMBIT, arXiv:1611.05065 [INSPIRE].

[13] J.A. Casas, D.G. Cerdeño, J.M. Moreno and J. Quilis, Reopening the Higgs portal for singlet scalar dark matter, arXiv:1701.08134 [INSPIRE].

[14] A. Beniwal et al., Combined analysis of effective Higgs portal dark matter models, Phys. Rev. D 93 (2016) 115016 [arXiv: 1512.06458] [INSPIRE].

[15] X.-G. He, T. Li, X.-Q. Li, J. Tandean and H.-C. Tsai, Constraints on scalar dark matter from direct experimental searches, Phys. Rev. D 79 (2009) 023521 [arXiv:0811.0658] [INSPIRE].

[16] A. Greljo, J. Julio, J.F. Kamenik, C. Smith and J. Zupan, Constraining Higgs mediated dark matter interactions, JHEP 11 (2013) 190 [arXiv:1309.3561] [INSPIRE].

[17] A. Drozd, B. Grzadkowski, J.F. Gunion and Y. Jiang, Extending two-Higgs-doublet models by a singlet scalar field - The case for dark matter, JHEP 11 (2014) 105 [arXiv:1408.2106] [INSPIRE].

[18] A. Drozd, B. Grzadkowski, J.F. Gunion and Y. Jiang, Isospin-violating dark-matter-nucleon scattering via two-Higgs-doublet-model portals, JCAP 10 (2016) 040 [arXiv:1510.07053] [INSPIRE].

[19] L. Wang and X.-F. Han, A simplified 2HDM with a scalar dark matter and the galactic center gamma-ray excess, Phys. Lett. B 739 (2014) 416 [arXiv:1406.3598] [INSPIRE].

[20] X.-G. He, B. Ren and J. Tandean, Hints of standard model Higgs boson at the LHC and light dark matter searches, Phys. Rev. D 85 (2012) 093019 [arXiv:1112.6364] [INSPIRE].

[21] C. Bird, R.V. Kowalewski and M. Pospelov, Dark matter pair-production in $b \rightarrow s$ transitions, Mod. Phys. Lett. A 21 (2006) 457 [hep-ph/0601090] [INSPIRE].

[22] X.-G. He, T. Li, X.-Q. Li and H.-C. Tsai, Scalar dark matter effects in Higgs and top quark decays, Mod. Phys. Lett. A 22 (2007) 2121 [hep-ph/0701156] [INSPIRE]. 
[23] B. Grzadkowski and P. Osland, Tempered two-Higgs-doublet model, Phys. Rev. D 82 (2010) 125026 [arXiv: 0910 .4068] [INSPIRE].

[24] M. Aoki, S. Kanemura and O. Seto, Multi-Higgs portal dark matter under the CDMS II results, Phys. Lett. B 685 (2010) 313 [arXiv:0912.5536] [INSPIRE].

[25] T. Li and Q. Shafi, Scalar dark matter search at the LHC through FCNC top decay, Phys. Rev. D 83 (2011) 095017 [arXiv:1101.3576] [INSPIRE].

[26] Y. Cai, X.-G. He and B. Ren, Low mass dark matter and invisible Higgs width in darkon models, Phys. Rev. D 83 (2011) 083524 [arXiv:1102.1522] [INSPIRE].

[27] Y. Bai, V. Barger, L.L. Everett and G. Shaughnessy, Two-Higgs-doublet-portal dark-matter model: LHC data and Fermi-LAT 135 GeV line, Phys. Rev. D 88 (2013) 015008 [arXiv: 1212.5604] [INSPIRE].

[28] X.-G. He and J. Tandean, Low-mass dark-matter hint from CDMS II, Higgs boson at the LHC and darkon models, Phys. Rev. D 88 (2013) 013020 [arXiv: 1304.6058] [INSPIRE].

[29] N. Okada and O. Seto, Galactic Center gamma-ray excess from two-Higgs-doublet-portal dark matter, Phys. Rev. D 90 (2014) 083523 [arXiv: 1408.2583] [INSPIRE].

[30] R. Campbell, S. Godfrey, H.E. Logan, A.D. Peterson and A. Poulin, Implications of the observation of dark matter self-interactions for singlet scalar dark matter, Phys. Rev. D 92 (2015) 055031 [arXiv: 1505.01793] [INSPIRE].

[31] X. Gao, Z. Kang and T. Li, Origins of the isospin violation of dark matter interactions, JCAP 01 (2013) 021 [arXiv:1107.3529] [INSPIRE].

[32] Y. Cai and T. Li, Singlet dark matter in a type-II two Higgs doublet model, Phys. Rev. D 88 (2013) 115004 [arXiv:1308.5346] [INSPIRE].

[33] A. Dutta Banik and D. Majumdar, Extension of minimal fermionic dark matter model: a study with two Higgs doublet model, Eur. Phys. J. C 75 (2015) 364 [arXiv:1311.0126] [INSPIRE].

[34] M.T. Frandsen, F. Kahlhoefer, S. Sarkar and K. Schmidt-Hoberg, Direct detection of dark matter in models with a light $Z^{\prime}$, JHEP 09 (2011) 128 [arXiv:1107.2118] [INSPIRE].

[35] G. Bélanger, A. Goudelis, J.-C. Park and A. Pukhov, Isospin-violating dark matter from a double portal, JCAP 02 (2014) 020 [arXiv:1311.0022] [INSPIRE].

[36] N. Chen, Q. Wang, W. Zhao, S.-T. Lin, Q. Yue and J. Li, Exothermic isospin-violating dark matter after SuperCDMS and CDEX, Phys. Lett. B 743 (2015) 205 [arXiv:1404.6043] [INSPIRE].

[37] C.-Q. Geng, D. Huang, C.-H. Lee and Q. Wang, Direct detection of exothermic dark matter with light mediator, JCAP 08 (2016) 009 [arXiv: 1605.05098] [INSPIRE].

[38] A. Kurylov and M. Kamionkowski, Generalized analysis of weakly interacting massive particle searches, Phys. Rev. D 69 (2004) 063503 [hep-ph/0307185] [INSPIRE].

[39] F. Giuliani, Are direct search experiments sensitive to all spin-independent WIMP candidates?, Phys. Rev. Lett. 95 (2005) 101301 [hep-ph/0504157] [INSPIRE].

[40] J.L. Feng, J. Kumar, D. Marfatia and D. Sanford, Isospin-violating dark matter, Phys. Lett. B 703 (2011) 124 [arXiv: 1102.4331] [INSPIRE].

[41] J.L. Feng, J. Kumar and D. Sanford, Xenophobic dark matter, Phys. Rev. D 88 (2013) 015021 [arXiv: 1306.2315 ] [INSPIRE]. 
[42] S. Chang, J. Liu, A. Pierce, N. Weiner and I. Yavin, CoGeNT interpretations, JCAP 08 (2010) 018 [arXiv: 1004.0697] [INSPIRE].

[43] C.E. Yaguna, Isospin-violating dark matter in the light of recent data, Phys. Rev. D 95 (2017) 055015 [arXiv: 1610.08683] [INSPIRE].

[44] Y.G. Kim and K.Y. Lee, The minimal model of fermionic dark matter, Phys. Rev. D 75 (2007) 115012 [hep-ph/0611069] [INSPIRE].

[45] I. Low, P. Schwaller, G. Shaughnessy and C.E.M. Wagner, The dark side of the Higgs boson, Phys. Rev. D 85 (2012) 015009 [arXiv:1110.4405] [INSPIRE].

[46] A. De Simone, G.F. Giudice and A. Strumia, Benchmarks for dark matter searches at the LHC, JHEP 06 (2014) 081 [arXiv: 1402.6287] [INSPIRE].

[47] S. Matsumoto, S. Mukhopadhyay and Y.-L.S. Tsai, Effective theory of WIMP dark matter supplemented by simplified models: singlet-like Majorana fermion case, Phys. Rev. D 94 (2016) 065034 [arXiv: 1604.02230] [INSPIRE].

[48] S. Kanemura, S. Matsumoto, T. Nabeshima and N. Okada, Can WIMP dark matter overcome the nightmare scenario?, Phys. Rev. D 82 (2010) 055026 [arXiv:1005.5651] [INSPIRE].

[49] A. Djouadi, O. Lebedev, Y. Mambrini and J. Quevillon, Implications of LHC searches for Higgs-portal dark matter, Phys. Lett. B 709 (2012) 65 [arXiv:1112.3299] [INSPIRE].

[50] A. Djouadi, A. Falkowski, Y. Mambrini and J. Quevillon, Direct detection of Higgs-portal dark matter at the LHC, Eur. Phys. J. C 73 (2013) 2455 [arXiv:1205.3169] [INSPIRE].

[51] J.F. Kamenik and C. Smith, FCNC portals to the dark sector, JHEP 03 (2012) 090 [arXiv: 1111.6402] [INSPIRE].

[52] J.F. Kamenik and C. Smith, Could a light Higgs boson illuminate the dark sector?, Phys. Rev. D 85 (2012) 093017 [arXiv: 1201.4814] [INSPIRE].

[53] M.A. Fedderke, J.-Y. Chen, E.W. Kolb and L.-T. Wang, The fermionic dark matter Higgs portal: an effective field theory approach, JHEP 08 (2014) 122 [arXiv:1404.2283] [INSPIRE].

[54] L. Lopez-Honorez, T. Schwetz and J. Zupan, Higgs portal, fermionic dark matter and a standard model like Higgs at 125 GeV, Phys. Lett. B 716 (2012) 179 [arXiv:1203.2064] [INSPIRE].

[55] Y.G. Kim, K.Y. Lee and S. Shin, Singlet fermionic dark matter, JHEP 05 (2008) 100 [arXiv: 0803.2932] [INSPIRE].

[56] S. Baek, P. Ko and W.-I. Park, Search for the Higgs portal to a singlet fermionic dark matter at the LHC, JHEP 02 (2012) 047 [arXiv:1112.1847] [INSPIRE].

[57] S. Baek, P. Ko, W.-I. Park and E. Senaha, Vacuum structure and stability of a singlet fermion dark matter model with a singlet scalar messenger, JHEP 11 (2012) 116 [arXiv: 1209.4163] [INSPIRE].

[58] H.-C. Tsai and K.-C. Yang, Dark matter mass constrained by the relic abundance, direct detections and colliders, Phys. Rev. D 87 (2013) 115016 [arXiv:1301.4186] [INSPIRE].

[59] Y.G. Kim, K.Y. Lee, C.B. Park and S. Shin, Secluded singlet fermionic dark matter driven by the Fermi gamma-ray excess, Phys. Rev. D 93 (2016) 075023 [arXiv:1601.05089] [InSPIRE].

[60] N.F. Bell, G. Busoni and I.W. Sanderson, Self-consistent Dark Matter Simplified Models with an s-channel scalar mediator, JCAP 03 (2017) 015 [arXiv: 1612.03475] [INSPIRE]. 
[61] S. Baek, P. Ko and W.-I. Park, Invisible Higgs decay width vs. dark matter direct detection cross section in Higgs portal dark matter models, Phys. Rev. D 90 (2014) 055014 [arXiv: 1405.3530] [INSPIRE].

[62] ATLAS, CMS collaboration, Combined measurement of the Higgs boson mass in pp collisions at $\sqrt{s}=7$ and 8 TeV with the ATLAS and CMS experiments, Phys. Rev. Lett. 114 (2015) 191803 [arXiv: 1503.07589] [InSPIRE].

[63] LhC Higgs Cross Section Working Group collaboration, J.R. Andersen et al., Handbook of LHC Higgs Cross Sections: 3. Higgs Properties, arXiv:1307.1347 [INSPIRE], updates available at https://twiki.cern.ch/twiki/bin/view/LHCPhysics/ CERNYellowReportPageBR2014.

[64] G. Busoni, A. De Simone, J. Gramling, E. Morgante and A. Riotto, On the validity of the effective field theory for dark matter searches at the LHC, part II: complete analysis for the s-channel, JCAP 06 (2014) 060 [arXiv: 1402.1275] [INSPIRE].

[65] XENON collaboration, E. Aprile et al., Physics reach of the XENON1T dark matter experiment, JCAP 04 (2016) 027 [arXiv: 1512.07501] [INSPIRE].

[66] C.E. Aalseth et al., The DarkSide multiton detector for the direct dark matter search, Adv. High Energy Phys. 2015 (2015) 541362.

[67] LZ collaboration, D.S. Akerib et al., LUX-ZEPLIN (LZ) conceptual design report, arXiv: 1509.02910 [INSPIRE].

[68] J. Billard, L. Strigari and E. Figueroa-Feliciano, Implication of neutrino backgrounds on the reach of next generation dark matter direct detection experiments, Phys. Rev. D 89 (2014) 023524 [arXiv: 1307.5458] [INSPIRE].

[69] W. Rarita and J. Schwinger, On a theory of particles with half integral spin, Phys. Rev. 60 (1941) 61 [INSPIRE].

[70] Z.-H. Yu, J.-M. Zheng, X.-J. Bi, Z. Li, D.-X. Yao and H.-H. Zhang, Constraining the interaction strength between dark matter and visible matter: II. scalar, vector and spin-3/2 dark matter, Nucl. Phys. B 860 (2012) 115 [arXiv:1112.6052] [InSPIRE].

[71] R. Ding and Y. Liao, Spin 3/2 particle as a dark matter candidate: an effective field theory approach, JHEP 04 (2012) 054 [arXiv:1201.0506] [INSPIRE].

[72] K.G. Savvidy and J.D. Vergados, Direct dark matter detection: a spin 3/2 WIMP candidate, Phys. Rev. D 87 (2013) 075013 [arXiv:1211.3214] [InSPIRE].

[73] R. Ding, Y. Liao, J.-Y. Liu and K. Wang, Comprehensive constraints on a spin-3/2 singlet particle as a dark matter candidate, JCAP 05 (2013) 028 [arXiv: 1302.4034] [INSPIRE].

[74] N.D. Christensen et al., Simulating spin- $\frac{3}{2}$ particles at colliders, Eur. Phys. J. C 73 (2013) 2580 [arXiv: 1308.1668] [INSPIRE].

[75] S. Dutta, A. Goyal and S. Kumar, Anomalous X-ray galactic signal from $7.1 \mathrm{keV} \mathrm{spin-3/2}$ dark matter decay, JCAP 02 (2016) 016 [arXiv: 1509.02105] [INSPIRE].

[76] M.O. Khojali, A. Goyal, M. Kumar and A.S. Cornell, Minimal spin-3/2 dark matter in a simple s-channel model, Eur. Phys. J. C 77 (2017) 25 [arXiv: 1608.08958] [INSPIRE].

[77] O. Lebedev, H.M. Lee and Y. Mambrini, Vector Higgs-portal dark matter and the invisible Higgs, Phys. Lett. B 707 (2012) 570 [arXiv:1111.4482] [INSPIRE].

[78] T. Hambye, Hidden vector dark matter, JHEP 01 (2009) 028 [arXiv:0811.0172] [INSPIRE]. 
[79] S. Baek, P. Ko, W.-I. Park and E. Senaha, Higgs portal vector dark matter: revisited, JHEP 05 (2013) 036 [arXiv: 1212.2131] [InSPIRE].

[80] M. Duch, B. Grzadkowski and M. McGarrie, A stable Higgs portal with vector dark matter, JHEP 09 (2015) 162 [arXiv: 1506.08805] [INSPIRE].

[81] J.F. Gunion, H.E. Haber, G.L. Kane and S. Dawson, The Higgs hunter's guide, Westview Press, Colorado U.S.A. (2000).

[82] G.C. Branco, P.M. Ferreira, L. Lavoura, M.N. Rebelo, M. Sher and J.P. Silva, Theory and phenomenology of two-Higgs-doublet models, Phys. Rept. 516 (2012) 1 [arXiv:1106.0034] [INSPIRE].

[83] M.E. Peskin and T. Takeuchi, Estimation of oblique electroweak corrections, Phys. Rev. D 46 (1992) 381 [INSPIRE].

[84] W. Grimus, L. Lavoura, O.M. Ogreid and P. Osland, The oblique parameters in multi-Higgs-doublet models, Nucl. Phys. B 801 (2008) 81 [arXiv:0802.4353] [INSPIRE].

[85] Particle Data Group collaboration, C. Patrignani, Review of particle physics, Chin. Phys. C 40 (2016) 100001.

[86] P. Gondolo and G. Gelmini, Cosmic abundances of stable particles: improved analysis, Nucl. Phys. B 360 (1991) 145 [INSPIRE].

[87] E.W. Kolb and M. Turner, The Early Universe, Westview Press, Boulder U.S.A. (1990).

[88] G. Steigman, B. Dasgupta and J.F. Beacom, Precise relic WIMP abundance and its impact on searches for dark matter annihilation, Phys. Rev. D 86 (2012) 023506 [arXiv:1204.3622] [INSPIRE].

[89] Planck collaboration, P.A.R. Ade et al., Planck 2015 results. XIII. Cosmological parameters, Astron. Astrophys. 594 (2016) A13 [arXiv:1502.01589] [INSPIRE].

[90] S. Kanemura, T. Kasai and Y. Okada, Mass bounds of the lightest CP even Higgs boson in the two Higgs doublet model, Phys. Lett. B 471 (1999) 182 [hep-ph/9903289] [INSPIRE].

[91] S. Kanemura, T. Kubota and E. Takasugi, Lee-Quigg-Thacker bounds for Higgs boson masses in a two doublet model, Phys. Lett. B 313 (1993) 155 [hep-ph/9303263] [INSPIRE].

[92] A.G. Akeroyd, A. Arhrib and E.-M. Naimi, Note on tree level unitarity in the general two Higgs doublet model, Phys. Lett. B 490 (2000) 119 [hep-ph/0006035] [INSPIRE].

[93] J.F. Gunion and H.E. Haber, The CP conserving two Higgs doublet model: the approach to the decoupling limit, Phys. Rev. D 67 (2003) 075019 [hep-ph/0207010] [INSPIRE]. 